\title{
Diffusion Processes in Lead-Uranium Systems
}

\author{
G. J. WASSERBURG \\ California Institute of Technology, Pasadena, California
}

\begin{abstract}
The ratio $r_{\lambda}$ of radiogenic daughter to residual parent (with decay constant $\lambda$ ) was investigated for a continuous diffusion model for a diffusion coefficient that is an arbitrary function of time. A solution for simultaneous diffusion of both parent and daughter is given in closed form. A theory is also developed that describes the trajectories $r_{\lambda}, r_{\lambda^{\prime}}$ for paired decay schemes (with decay constants $\lambda$ and $\lambda^{\prime}$ ) in the neighborhood of concordia. A wide class of systems is shown to be linear in the $r_{\lambda}, r_{\lambda^{\prime}}$ diagram when $\int_{0}^{\tau} D(\eta) \boldsymbol{d}_{\eta} / a^{2} \ll 1$. A radiation damage model is proposed for the diffusion coefficient that relates $D(\tau)$ to the integrated irradiation damage and to the uranium (and thorium) contents. The functional relationship between $r_{\lambda}$ and the uranium content is found for three important cases. The model $D(\tau)=D_{1} \tau$ yields a $r_{\lambda}, r_{\lambda^{\prime}}$ curve that is very similar to that obtained by Tilton for $D=D_{0}$. The slope is somewhat greater and the linear region more extended. The theoretical curves are found to be in reasonable agreement with the existing experimental data, and they fit the existing data at least as well as the constant $D$ model.
\end{abstract}

Introduction. Wetherill [1956] considered the problem of lead loss and uranium loss or gain in systems due to episodic events. He showed that the ratios $\mathrm{Pb}^{206} / \mathrm{U}^{238}$ and $\mathrm{Pb}^{207} / \mathrm{U}^{235}$ for all systems of age $\tau_{0}$ that later undergo an episodic disturbance at time $\tau_{1}$ will lie on a straight line in a concordia diagram. This straight line will intersect the concordia curve (the parametric curve $\exp \left[\tau \lambda_{U}\right.$ ss $\left.]-1, \exp \left[\tau \lambda_{U_{\text {2ss }}}\right]-1\right)$ at the points corresponding to $\tau_{0}$ and $\tau_{1}$. By means of this model, a linear array of experimental results on uranium minerals from cogenetic rocks could thus be interpreted. The two intersections with the concordia curve correspond to the times of the primary and secondary events (Figure 1).

If we consider the loss of radiogenic daughter from a system due to diffusion with a fixed value for the diffusion constant $D_{0}$, we obtain an expression for the ratio $r_{\lambda}$ of the remaining daughter product to the parent nuclide in a given grain. This ratio $r_{\lambda}$ is a function of the time, the grain geometry, the diffusion coefficient, and the decay constant $(\lambda)$ of the parent. The special case for spherical geometry was solved by Wasserburg [1954]. Nicolaysen [1957] first considered this mechanism as a means of explaining discordances in lead-uranium systems. Using Wasserburg's model, Tilton [1960] showed that for $\mathrm{Pb}^{206} / \mathrm{U}^{238}$ and $\mathrm{Pb}^{207} / \mathrm{U}^{235}$ the functions $r_{\lambda}$ and $r_{\lambda}$, for samples of the same age $\tau$, but of different values of $D_{0} / a^{2}$, gave a simple curve on an $r_{\lambda}, r_{\lambda}$, diagram. This curve passes through the concordia curve at the points corresponding to $\tau$ and zero; it has a rather constant slope over a wide part of the diagram, but in the neighborhood of the origin the curvature increases markedly. When we use the episodic loss model, experimental points lying along the rather linear part of the curve give a correct primary event of age $\tau_{0}$ and a spurious event $\tau_{1}$ at the extrapolated lower intersection with the concordia curve (Figure 2). The primary age $\tau_{0}$ for the continuous diffusion model uniquely determines the extrapolated intercept $\tau_{1}$. Tilton showed that a wide variety of samples of approximately the same $\mathrm{Pb}^{206}-\mathrm{Pb}^{207}$ age lay along a diffusion trajectory, and that this held for a variety of ages. This discovery clarified the problem of an unidentified worldwide event at about $500 \mathrm{~m} . \mathrm{y}$. and the observation made by Russell and Ahrens [1957] that there was a regular relationship between the primary age of a linear array and the apparent secondary age of episodic loss.

The purpose of the present paper is to discuss the problem of diffusion discordance in a more general form and to suggest an alternate mechanism to Tilton's model. The diffusion problem can be generalized to the form:

$$
\begin{aligned}
& \frac{\partial}{\partial \tau} C_{\lambda}(\mathbf{r}, \tau) \\
& \quad=D(\tau) \nabla^{2} C_{\lambda}(\mathrm{r}, \tau)+\lambda U_{\lambda}(\mathrm{r}, \tau)
\end{aligned}
$$




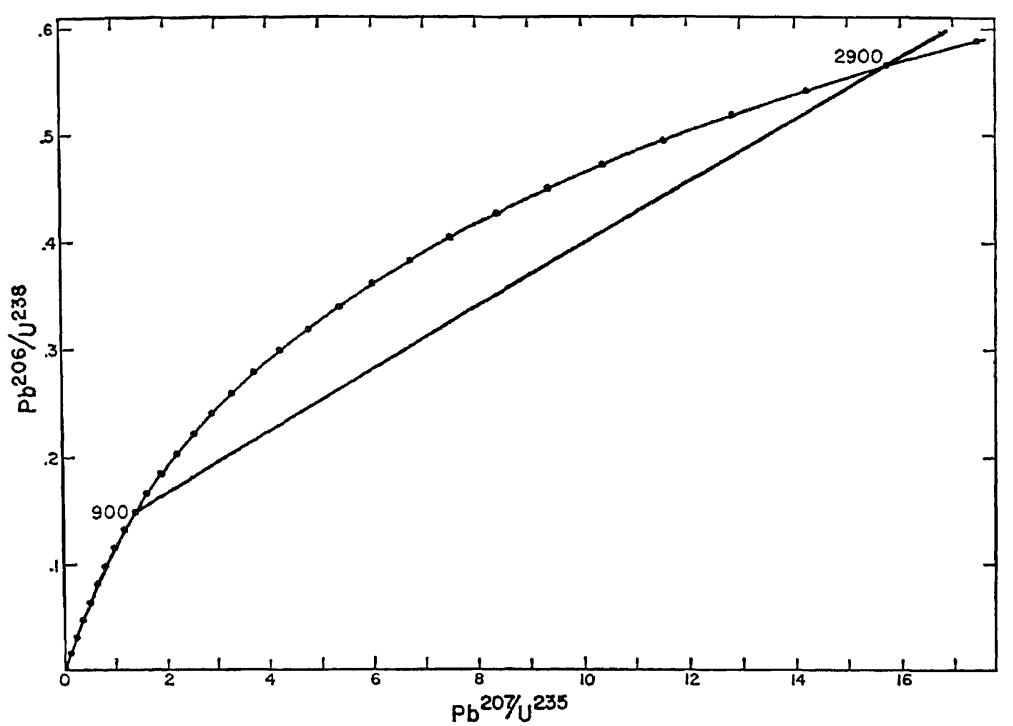

Fig. 1. Episodic loss chord for a primary age of 2900 m.y. and a secondary event at 900 m.y. The curve is the concordia curve $r_{\lambda}=e^{\lambda_{\tau}}-1, r_{\lambda^{\prime}}=e^{\lambda^{\prime} \tau}-1$ for $\mathrm{U}^{235}$ and $\mathrm{U}^{238}$, respectively. The dots correspond to values of $\tau$ in $100-m . y$. intervals.

$\frac{\partial U_{\lambda}(\mathbf{r}, \tau)}{\partial \tau}$

$$
=D^{*}(\tau) \nabla^{2} U_{\lambda}(\mathrm{r}, \tau)-\lambda U_{\lambda}(\mathrm{r}, \tau)
$$

with the initial condition $C_{\lambda}(\mathrm{r}, 0)=0$. The boundary condition is taken as $C_{\lambda}(r, \tau)=0$ on a specified surface $\sigma$. Here $U_{\lambda}$ is the concentration of the radioactive parent, $C_{\lambda}$ is the concentration of the stable daughter product, and $\lambda$ is the decay constant of $U_{\lambda} . D(\tau)$ and $D^{*}(\tau)$ are the diffusion coefficients of daughter and parent, respectively. The ratio of daughter product to

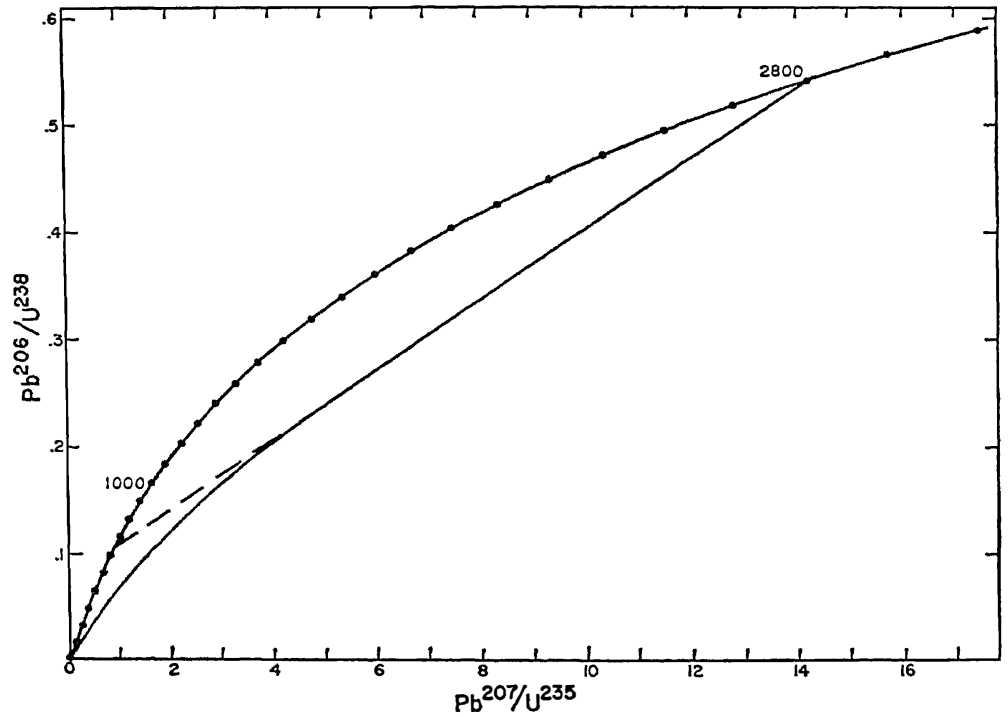

Fig. 2. Continuous diffusion trajectory for primary age of 2800 m.y. for the model $D(\tau)=$ $D_{0}$ (a constant). The dashed line indicates the extrapolation of the linear part of the diffusion trajectory. 
parent at time $\tau$ in the volume $V$ enclosed by $\sigma$ is $r_{\lambda}$.

$r_{\lambda}=\int_{V} C_{\lambda}(\mathbf{r}, \tau) d V / \int_{V} \mathrm{U}_{\lambda}(\mathbf{r}, \tau) d V$

For $\mathrm{Pb}^{206} / \mathrm{U}^{238}$ and $\mathrm{Pb}^{207} / \mathrm{U}^{235}$ there are two similar equations. Since both the parents and daughters are isotopes of the same two elements, it is reasonable to assume the diffusion coefficients to be identical. The present discussion ignores the problem of losses in the chain of intermediate daughters. We will first discuss the case in which the concentration $U_{\lambda}$ is homogeneous and in which the change in $U_{\lambda}$ is only due to radioactive decay. Hence $U_{\lambda}=U_{\lambda}{ }^{0} e^{-\lambda \tau}$.

For this case solutions can be obtained for a variety of geometries (see appendix). For a sphere of radius $a$

$$
\begin{aligned}
& r_{\lambda}=r_{\lambda}\left(\tau,\left[D(\tau) / a^{2}\right]\right)=\frac{6 \lambda}{\pi^{2}} \sum_{n=1}^{\infty} \frac{1}{n^{2}} \\
& \int_{0}^{\tau} \exp \left\{-n^{2} \pi^{2}[T(\tau)-T(\mu)] / a^{2}+\lambda(\tau-\mu)\right\} d \mu
\end{aligned}
$$

where $T(\tau)=\int_{0} \tau D(\eta) d \eta$. We have written $r_{\lambda}$ in the second form to emphasize that it depends on $\lambda, \tau$, and the function $D(\tau) / a^{2}$. In this paper we will use $r_{\lambda^{\prime}}$ and $r_{\lambda}$ to denote the $\mathrm{Pb}^{206} / \mathrm{U}^{238}$ and $\mathrm{Pb}^{207} / \mathrm{U}^{235}$ ratios, respectively. For a given function $T(\mu)(\mu \leq \tau)$ and a fixed $\tau$, the functions $r_{\lambda^{\prime}}$ and $r_{\lambda}$ define a curve for the parametric variable $T(\mu) / a^{2}$. This curve will be referred to as a diffusion trajectory. If $D(\tau)$ does not have any singularities, the curve will be called a continuous diffusion trajectory. The formal calculations can of course be used for other decay schemes where the assumptions are valid. Tilton, in his discussion of the case $D(\tau)=D_{0}$ (a constant), pointed out some difficulties. One of these is that, since the activation energy for diffusion is usually high, it would be expected that temperature fluctuations over geologic time would cause natural systems of a given age to fall off the curve $r_{\lambda}\left(\tau, D_{0} / a^{2}\right), r_{\lambda^{\prime}}\left(\tau, D_{0} / a^{2}\right)$. In addition, he pointed out that sometimes radiation-produced vacancies will not be temperature-dependent and that the degree of metamictization could affect the diffusion process. - Tilton also pointed out that the diffusion coeffi- cient might increase with the amount of bombardment, but indicated that this would greatly affect the resulting curves. In the following sections we will show that considerable variation of $D$ with $\tau$ will not greatly affect the nature of the curve and that a model of radiation damage will only alter the results by a small amount that is consistent with the existing data.

Radiation damage model. The experimental data used by Tilton in his volume diffusion model were from suites of rocks with approximately the same $\mathrm{Pb}^{207} / \mathrm{Pb}^{206}$ age. This comparison showed quite clearly that relatively uniform diffusion must be the explanation in many cases of discordance. A more detailed analysis was not possible because the minerals used were not all of the same primary age. A careful study was made by Silver and Deutsch [1961] on a number of zircon separates from an igneous rock. These different zircon fractions plotted on a straight line on the $\mathrm{Pb}^{208} / \mathrm{U}^{238}, \mathrm{~Pb}^{207} / \mathrm{U}^{205}$ diagram. As was pointed out by Silver, such studies on different zircon fractions from a particular igneous rock permit a more detailed understanding of the loss processes since the assumption that the zircons are cogenetic can be justified.

Using a suite of uranium minerals from the Johnny Lyon granodiorite, Silver and Deutsch obtained a linear array that was not in agreement with a continuous diffusion model, but that indicated an episodic event at 90 m.y. and a primary age of 1660 m.y. The local geological relationships strongly supported such an interpretation. This result clearly indicated that some discordances are dominated by the affects of episodic disturbances and are not due to continuous diffusion loss on a long time scale.

Later work by Silver and McKinney [1962] on a granite from the Marble Mountains in the Mojave Desert gave a linear array for several zircon fractions. On the basis of an episodic loss model, these data indicated an episodic loss at 180 m.y. and a primary age of 1450 m.y. The experimental data were displaced a small but distinct amount from the $1450 \mathrm{~m} . \mathrm{y}$. curve for Tilton's model, the lower intercept being below that obtained for Tilton's curve. Silver (personal communication) also found a correlation between the uranium concentration and degree of discordance for zircons from this locality. Geochronologic and field work by Lanphere [1962] on the same granite showed no evidence for a 
significant younger event from consideration of the $\mathrm{A}^{40}-\mathrm{K}^{40}$ and $\mathrm{Sr}^{87}-\mathrm{Rb}^{87}$ ages on biotites and feldspars.

These observations suggested that it would be valuable to investigate the formal problem for a time-dependent diffusion coefficient and, in particular, to estimate the functional relationship between $D(\tau)$ and the irradiation history. The relationships observed by Silver [1962] between uranium concentration and degree of discordance were particularly important in stimulating this work. The total irradiation a grain receives over a period $\tau$ due to uranium, thorium decay is of the form

$$
\begin{aligned}
A U^{238}(\tau)[\exp & \left.\left(\tau \lambda_{\mathrm{U}^{23 \mathrm{a}}}\right)-1\right] \\
& +B \mathrm{U}^{235}(\tau)\left[\exp \left(\tau \lambda_{\mathrm{U}^{23 \mathrm{~s}}}\right)-1\right] \\
& +C \operatorname{Th}^{232}(\tau)\left[\exp \left(\tau \lambda_{\mathrm{Th}^{23}}\right)-1\right]
\end{aligned}
$$

For low dosages the radiation damage is proportional to the irradiation, and the numbers $A$, $B$, and $C$ are then constants depending on the number, energy, and type of particles emitted in each decay series and the mineral species. Since the decay schemes are similar, and since the major effect must be due to the $\alpha$ particles, the ratios must be approximately $A: B: C:: 8: 7: 6$. Because of the low abundance of $U^{235}$ today, the major effects are due to the $\mathrm{U}^{238}$ and $\mathrm{Th}^{232}$ decays. Since both these elements have long half-lives, it is reasonable to take the total radiation damage as proportional to the time $\tau$. For the extreme case when no thorium is present, the departure from linearity is only about 15 per cent at $2000 \mathrm{~m}$. y. If the radiation is very large or at relatively elevated temperatures, there is the possibility of self annealing and saturation of the induced defects, and the radiation damage would be approximately of the form $\left(D_{1} / b\right)\left(1-e^{-b \tau}\right)$. Let us take the diffusion coefficient to be proportional to the radiation damage. If the defects have a relatively high mobility so that the number density of defects becomes saturated over a short time scale, $D(\tau)$ will be proportional to the irradiation rate. This will also be a rather complicated function of time because of the explicit formula for the total irradiation rate. For simplicity we can consider the case for a constant rate of irradiation $\left(\lambda^{\prime} \tau \ll 1\right)$; then $D(\tau)$ will be a constant. This constant will be proportional to the rate of production of defects and hence to the uranium, thorium concentrations. This corresponds in the isothermal diffusion case $D(\tau)=D_{0}=D_{\mathrm{i}} / \mathrm{b}$, and the curves given by Tilton will apply. In the region where the damage is not saturated we will assume it to be proportional to the integrated irradiation. The correlation existing between specific radiation damage and age suggests that the proportionality relationship is reasonable. However, the numerous failures of the radiation damage age method indicate that this cannot be taken as a general relationship. In the present discussion we will assume that the damage is proportional to the integrated irradiation. There is unfortunately no known relationship between the diffusion coefficient and the irradiation. Some discussion of radiation-enhanced diffusion has been given by Dienes and Damask [1958, 1962]. A discussion of $\mathrm{He}^{4}$ loss from minerals due to radiation damage was given by Hurley [1954]. The arguments for $D(\tau)$ given here are to some extent similar to those presented previously by Hurley for the rate constant in his treatment. A plausible assumption is that the number density of lattice defects is proportional to the irradiation and that these defects are the principle diffusion centers. Hence $D(\tau)=D_{1} \tau$, where $D_{1}$ is a constant. $D_{1}$ therefore must be proportional to $\mathrm{U}_{0}{ }^{238}+0.23 \mathrm{Th}_{0}{ }^{232}$.

We will assume here that the intrinsic coeffcient of diffusion in the absence of irradiation $\left(D_{0}\right)$ is negligible in comparison with the effective value of $D_{1} \tau$ and will investigate the case $D(\tau)=D_{1} \tau$. The temperature dependence of $D_{1}$ must be considerably less than that of $D_{0}$, since the number of defect sites is no longer a function of temperature. For the case of spherical geometry, the function $r_{\lambda}$ is obtained by substitution in (3). Values of $r_{\lambda^{\prime}}$ and $r_{\lambda}$ for $\mathrm{Pb}^{206 / \mathrm{U}^{238}}$ and $\mathrm{Pb}^{207} / \mathrm{U}^{235}$ are given in Table 1 for different values of $\tau$ and $K\left(K=\pi \sqrt{2 D_{1}} / a\right)$. In addition, the asymptotic slopes $d r_{\lambda} / d r_{\lambda^{\prime}}$ in the limit as $K \tau \ll 2 \pi$ are given in Table $2 a$ (see appendixes). The asymptotic slopes $d r_{\lambda} / d r_{\lambda^{\prime}}$ for the case $D(\tau)=D_{0}$ are given in Table $2 b$. These slopes are a good approximation to the slopes of the straight part of the diffusion trajectories and are equal to the tangent of the diffusion trajectory at the intersection with concordia. By comparison it is evident that the slopes for the irradiation case correspond to an extrapolated intercept on the concordia curve which represents a younger spurious second event than 
Table 1 Tabulated Values of $r_{\lambda^{\prime}}$ and $x_{\lambda}$ for Different Primary Ages and Diffusion Parameter $(K) . \quad \lambda^{\prime}=1.54 \times 10^{-10} y^{-1}$. $\lambda=9.72 \times 10^{-1} y^{-1}$. The first row for each primary age is $r_{\lambda}$ and the second row is $r_{\lambda}$. E $\pm x$ stands for $10 \pm x$.

\begin{tabular}{|c|c|c|c|c|c|c|c|c|c|c|}
\hline $\begin{array}{l}\text { Primary } \\
\text { Age (AB) }\end{array}$ & $\begin{array}{c}K \times 10^{10} \\
1.0\end{array}$ & 1.75 & 2.0 & 2.75 & 3.5 & 4.0 & 5.0 & 6.0 & 6.75 & 7.5 \\
\hline 1.0 & $\begin{array}{l}1.5942 \mathrm{E}-1 \\
1.5696\end{array}$ & $\begin{array}{l}1.5426 \mathrm{E}-1 \\
1.5158\end{array}$ & $\begin{array}{l}1.5256 \mathrm{E}-1 \\
1.4981\end{array}$ & $\begin{array}{l}1.4753 \mathrm{E}-1 \\
1.4457\end{array}$ & $\begin{array}{l}1.4259 \mathrm{E}-1 \\
1.3944\end{array}$ & $\begin{array}{l}1.3935 \mathrm{E}-1 \\
1.3607\end{array}$ & & & $8^{0 E-1}$ & $\begin{array}{l}787 E-1 \\
380\end{array}$ \\
\hline 1.1 & $\begin{array}{l}1.7598 \mathrm{E}-1 \\
1.8183\end{array}$ & $\begin{array}{l}1.6970 \mathrm{E}-1 \\
1.7493\end{array}$ & $\begin{array}{l}1.6763 \mathrm{E}-1 \\
1.7267\end{array}$ & $\begin{array}{l}1.6152 \mathrm{E}-1 \\
1.6596\end{array}$ & $\begin{array}{l}1.5554 \mathrm{E}-1 \\
1.5940\end{array}$ & $\begin{array}{l}1.5162 \mathrm{E}-1 \\
1.5511\end{array}$ & $\begin{array}{l}1.4 \\
1.4\end{array}$ & $1^{4 E-1}$ & $9^{2 B-1}$ & $\begin{array}{l}1.2583 \mathrm{E}-1 \\
1.2693\end{array}$ \\
\hline 3.2 & $\begin{array}{l}1.9265 \mathrm{E}-1 \\
2.0906\end{array}$ & $\begin{array}{l}1.8513 \mathrm{E}-1 \\
2.0034\end{array}$ & $\begin{array}{l}1.8266 \mathrm{E}-1 \\
1.9748\end{array}$ & $\begin{array}{l}1.7537 \mathrm{E}-1 \\
1.8904\end{array}$ & $\begin{array}{l}1.6824 \mathrm{E}-1 \\
1.8080\end{array}$ & $\begin{array}{l}1.6359 \mathrm{E}-1 \\
1.7542\end{array}$ & $\begin{array}{l}1.5451 \mathrm{E}-1 \\
1.6493\end{array}$ & $\begin{array}{l}1.4572 \mathrm{E}-1 \\
1.5482\end{array}$ & $\begin{array}{l}1.3934 \mathrm{E}-1 \\
1.4747\end{array}$ & $\begin{array}{l}1.3312 \mathrm{E}-1 \\
1.4033\end{array}$ \\
\hline 1.3 & $\begin{array}{l}2.0943 \mathrm{E}-1 \\
2.3885\end{array}$ & $\begin{array}{l}2.0056 \mathrm{E}-1 \\
2.2799\end{array}$ & $\begin{array}{l}1.9765 \mathrm{E}-1 \\
2.2443\end{array}$ & $\begin{array}{l}1.8907 \mathrm{E}-1 \\
2.1393\end{array}$ & $\begin{array}{l}1.8070 \mathrm{E}-1 \\
2.0372\end{array}$ & $\begin{array}{l}1.7524 \mathrm{E}-1 \\
1.9707\end{array}$ & $\begin{array}{l}1.6462 \mathrm{E}-1 \\
1.8413\end{array}$ & $\begin{array}{l}1.5439 \mathrm{E}-1 \\
1.7170\end{array}$ & $\begin{array}{l}1.4697 \mathrm{E}-1 \\
1.6270\end{array}$ & $\begin{array}{l}1.3976 \mathrm{E}-1 \\
1.5398\end{array}$ \\
\hline 1.4 & $\begin{array}{l}2.2633 \mathrm{E}-1 \\
2.7145\end{array}$ & $\begin{array}{l}2.1598 \mathrm{E}-1 \\
2.5807\end{array}$ & $\begin{array}{l}2.1260 \mathrm{E}-1 \\
2.5369\end{array}$ & $\begin{array}{l}2.0261 \mathrm{E}-1 \\
2.4080\end{array}$ & $\begin{array}{l}1.9290 \mathrm{E}-1 \\
2.2828\end{array}$ & $\begin{array}{l}1.8658 E-I \\
2.2014\end{array}$ & $\begin{array}{l}1.7431 \mathrm{E}-1 \\
2.0437\end{array}$ & $\begin{array}{l}1.6253 \mathrm{E}-1 \\
1.8926\end{array}$ & $\begin{array}{l}1.5401 \mathrm{E}-1 \\
1.7837\end{array}$ & $\begin{array}{l}1.4577^{\mathrm{E}-1} \\
1.6785\end{array}$ \\
\hline 1.45 & $\begin{array}{l}2.3483 E-1 \\
2.8889\end{array}$ & $\begin{array}{l}2.2370 \mathrm{E}-1 \\
.2 .7409\end{array}$ & $\begin{array}{l}2.20 \\
2.69\end{array}$ & $\begin{array}{l}2.093 \\
2.550\end{array}$ & $\begin{array}{l}1.9891 E-1 \\
2.4121\end{array}$ & $\begin{array}{l}1.9213 \mathrm{E}-1 \\
2.3225\end{array}$ & $\begin{array}{l}1.7899 \mathrm{E}-1 \\
2.1489\end{array}$ & $\begin{array}{l}1.6640 \mathrm{E}-1 \\
1.9831\end{array}$ & $E-1$ & $\begin{array}{l}1.4854 \mathrm{E}-1 \\
1.7486\end{array}$ \\
\hline 1.6 & $\begin{array}{l}2.6048 \mathrm{E}-1 \\
3.4619\end{array}$ & $\begin{array}{l}2.4681 \mathrm{E}-1 \\
3.2643\end{array}$ & $\begin{array}{l}2.4235 \mathrm{E}-1 \\
3.1998\end{array}$ & $\begin{array}{l}2.292 \\
3.010\end{array}$ & $\begin{array}{l}2.1654 \mathrm{E}-1 \\
2.8279\end{array}$ & $\begin{array}{l}2.0831 E-1 \\
2.7096\end{array}$ & $\begin{array}{l}1.9241 \mathrm{E}-1 \\
2.4816\end{array}$ & $\begin{array}{l}1.7726 \mathrm{E}-1 \\
2.2650\end{array}$ & $\begin{array}{l}1.6638 \mathrm{E}-1 \\
2.1100\end{array}$ & $\begin{array}{l}1.5592 \mathrm{E}-1 \\
1.9615\end{array}$ \\
\hline 1.7 & $\begin{array}{l}2.7773 E-1 \\
3.8894\end{array}$ & $\begin{array}{l}2.6221 \mathrm{E}-1 \\
3.6520\end{array}$ & $5 E-1$ & $\begin{array}{l}2.42 \\
3.34\end{array}$ & $\begin{array}{l}2.2797 \mathrm{E}-1 \\
3.1299\end{array}$ & $\begin{array}{l}2.1870 \mathrm{E}-1 \\
2.9889\end{array}$ & $\begin{array}{l}2.0082 \mathrm{E}-\mathrm{I} \\
2.7181\end{array}$ & $\begin{array}{l}1.8386 \mathrm{E}-1 \\
2.4618\end{array}$ & $\begin{array}{l}1.7172 \mathrm{E}-1 \\
2.2793\end{array}$ & $\begin{array}{l}1.6010 \mathrm{E}-1 \\
2.1050\end{array}$ \\
\hline 1.8 & $2.9510 \mathrm{E}-1$ & $\begin{array}{l}2.7761 \mathrm{E}-1 \\
4.0740\end{array}$ & $\begin{array}{l}2.71 \\
3.98\end{array}$ & $2-1$ & $\begin{array}{l}2.3914 \mathrm{E}-1 \\
3.4534\end{array}$ & $\begin{array}{l}2.2876 \mathrm{E}-1 \\
3.2865\end{array}$ & $\begin{array}{l}2.0882 \mathrm{E}-1 \\
2.9669\end{array}$ & $\begin{array}{l}1.8995 \mathrm{E}-1 \\
2.6658\end{array}$ & $3^{1 E-1}$ & $\begin{array}{l}1.6369 \mathrm{E}-1 \\
2.2493\end{array}$ \\
\hline 1.9 & $\begin{array}{l}3.1258 \mathrm{E}-1 \\
4.8697\end{array}$ & $\begin{array}{l}2.9298 \mathrm{E}-1 \\
4.5334\end{array}$ & $\begin{array}{l}2.86 \\
4.42\end{array}$ & $\begin{array}{l}2.6797 \mathrm{E}-1 \\
4.1054\end{array}$ & $\begin{array}{l}2.500 \\
3.790\end{array}$ & $E-1$ & $\begin{array}{l}2.1638 \mathrm{E}-1 \\
3.2285\end{array}$ & $E-1$ & $B^{7 E-1}$ & $\begin{array}{l}1.6671 \mathrm{E}-1 \\
2.3938\end{array}$ \\
\hline 2.0 & $\begin{array}{l}3019 E-1 \\
4305\end{array}$ & $\begin{array}{l}3.0835 \mathrm{E}-1 \\
5.0334\end{array}$ & -1 & $\begin{array}{l}2.8055 \mathrm{E}-1 \\
4.5295\end{array}$ & $\begin{array}{l}2.6069 \mathrm{E}-1 \\
4.1709\end{array}$ & $E-1$ & $\begin{array}{l}2.2353 \mathrm{E}-1 \\
3.5034\end{array}$ & $E-1$ & E-1 & $\begin{array}{l}9 E-1 \\
2\end{array}$ \\
\hline 2.1 & $\begin{array}{l}4792 E-1 \\
0445\end{array}$ & $\begin{array}{l}3.237 \\
5.577\end{array}$ & $\begin{array}{l}3.1 \\
5.4\end{array}$ & $\begin{array}{l}2.9296 \mathrm{E}-1 \\
4.9870\end{array}$ & $E-1$ & $3^{2 E-1}$ & $\begin{array}{l}2.3 \\
3.7\end{array}$ & $3 E-1$ & $7^{1 E-1}$ & $\begin{array}{l}1.71 \\
2.68\end{array}$ \\
\hline 2.2 & $\begin{array}{l}6576 \mathrm{E}-1 \\
7167\end{array}$ & $\begin{array}{l}3.39 \\
6.17\end{array}$ & -1 & $\begin{array}{l}3.05 \\
5.48\end{array}$ & -1 & $2-1$ & $\begin{array}{l}2.3 \\
4.0\end{array}$ & & $\frac{2 E-1}{4}$ & $5^{7 E-1}$ \\
\hline 2.3 & $\begin{array}{l}8373 E-1 \\
4525\end{array}$ & -1 & $E-1$ & & & $E-1$ & & & $E-1$ & E-1 \\
\hline 2.4 & $\begin{array}{l}\text { 0182E-1 } \\
2582\end{array}$ & -1 & & $E-1$ & & $\begin{array}{l}.8235 E-1 \\
.5220\end{array}$ & & & $E-1$ & $\begin{array}{l}9 \mathrm{E}-1 \\
5\end{array}$ \\
\hline 2.5 & $\begin{array}{l}2003 E-1 \\
1403\end{array}$ & $\begin{array}{l}3494 E-1 \\
2813\end{array}$ & 52 & $\begin{array}{l}4086 \mathrm{E}-1 \\
2079\end{array}$ & $8^{5 E-1}$ & 4 & $E-1$ & $\begin{array}{l}1 E-1 \\
0\end{array}$ & $3-1$ & $2 E-1$ \\
\hline 2.6 & $\begin{array}{l}3836 \mathrm{E}-1 \\
0106 \mathrm{E}+1\end{array}$ & $\begin{array}{l}019 \mathrm{E}-1 \\
133\end{array}$ & $\begin{array}{l}791 E-1 \\
948\end{array}$ & . & $\begin{array}{l}886 E-1 \\
143\end{array}$ & $E-1$ & $\begin{array}{l}76 \mathrm{E}-1 \\
81\end{array}$ & & & $\begin{array}{l}7362 E-1 \\
3627\end{array}$ \\
\hline 2.7 & $\begin{array}{l}5682 R-1 \\
1164 E+1\end{array}$ & $\begin{array}{l}-1 \\
+1\end{array}$ & $38-1$ & 1 & -1 & $E-1$ & -1 & & & 1.7 \\
\hline 2.8 & $\begin{array}{l}7540 \mathrm{E}-1 \\
2322 \mathrm{E}+1\end{array}$ & $\begin{array}{l}-1 \\
+1\end{array}$ & $\begin{array}{l}-1 \\
+1\end{array}$ & .7 & $3^{4 E-1}$ & 2 & $E-1$ & & & -1 \\
\hline 2.9 & $\begin{array}{l}941 \text { IE }-1 \\
3589 E+1\end{array}$ & $\begin{array}{l}-1 \\
+1\end{array}$ & -1 & $\begin{array}{l}1 \\
-1\end{array}$ & $1 E-1$ & $\begin{array}{l}2 E-1 \\
4\end{array}$ & -1 & & & -1 \\
\hline 3.0 & $\begin{array}{l}1294 E-1 \\
4978 E+1\end{array}$ & $\begin{array}{l}-1 \\
+1\end{array}$ & $\begin{array}{l}-1 \\
-1\end{array}$ & 1 & $0^{O E-1}$ & $2^{7 E-1}$ & $8^{6 E-1}$ & E-1 & & -1 \\
\hline 3.1 & $\begin{array}{l}3189 \mathrm{E}-1 \\
6497 \mathrm{E}+1\end{array}$ & $\begin{array}{l}-1 \\
1-1\end{array}$ & $\begin{array}{l}-1 \\
-2\end{array}$ & 1 & $\begin{array}{l}-1 \\
+1\end{array}$ & $E-1$ & -1 & & & \\
\hline 3.2 & $\begin{array}{l}-1 \\
+1\end{array}$ & 1 & $\begin{array}{l}1 \\
1\end{array}$ & 1 & & -1 & & $8-1$ & $E-1$ & $E-1$ \\
\hline 3.3 & $\begin{array}{l}-1 \\
+1\end{array}$ & $\begin{array}{l}-1 \\
+1\end{array}$ & 1. & $\begin{array}{l}4.2782 \mathrm{E}-1 \\
1.4276 \mathrm{E}+1\end{array}$ & & $\begin{array}{l}1 \\
-1\end{array}$ & & -1 & & $E-1$ \\
\hline 3.4 & $\begin{array}{l}-1 \\
+1\end{array}$ & $\begin{array}{l}-1 \\
+1\end{array}$ & $\begin{array}{l}-1 \\
+1\end{array}$ & 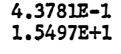 & & $\begin{array}{l}-1 \\
+1\end{array}$ & & -1 & $E-1$ & $2 E-1$ \\
\hline 4.4 & $\begin{array}{l}9007 \mathrm{E}-1 \\
5975 \mathrm{E}+1\end{array}$ & $\begin{array}{l}6.6933 E-1 \\
4.5952 \mathrm{E}+1\end{array}$ & $4.2849 \mathrm{E}+1$ & 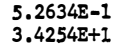 & & 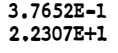 & & & & \\
\hline 4.5 & $389 E+1$ & $\begin{array}{l}6.8387 \mathrm{E}-1 \\
5.0110 \mathrm{E}+1\end{array}$ & $\begin{array}{l}.4432 \mathrm{E}-1 \\
.6626 \mathrm{E}+1\end{array}$ & 0 & & 等 & & $\begin{array}{l}.0010 E-1 \\
.5601\end{array}$ & $\begin{array}{l}517 E-1 \\
3670\end{array}$ & $\begin{array}{l}1.2050 \mathrm{E}-1 \\
4.1193\end{array}$ \\
\hline 4.6 & $7316 \mathrm{E}+1$ & $4633 E+1$ & $5.0723 \mathrm{E}+1$ & .995 & $613 E+1$ & 2.5 & $8-1$ & $E-1$ & 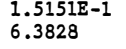 & 4.05 \\
\hline
\end{tabular}


Table I (Continued)

\begin{tabular}{|c|c|c|c|c|c|c|c|c|c|c|}
\hline $\begin{array}{l}\text { Primary } \\
\text { Age ( } \Delta E)\end{array}$ & $\begin{array}{c}\mathrm{K} \times 10^{10} \\
8.0\end{array}$ & 10.5 & 12.5 & 14.0 & 14.75 & 15.5 & 16.0 & 21.0 & 25.0 & 28.0 \\
\hline 1.0 & $\begin{array}{l}1.1497 \mathrm{E}-1 \\
1.1081\end{array}$ & $\begin{array}{l}1.0114 \mathrm{E}-1 \\
9.6523 \mathrm{E}-1\end{array}$ & $\begin{array}{l}9.0838 \mathrm{E}-2 \\
8.5933 \mathrm{E}-1\end{array}$ & $\begin{array}{l}8.3566 \mathrm{E}-2 \\
7.8477 \mathrm{E}-1\end{array}$ & $\begin{array}{l}8.0076 \mathrm{E}-2 \\
7.4907 \mathrm{E}-1\end{array}$ & $\begin{array}{l}7.6681 \mathrm{E}-2 \\
7.1441 \mathrm{E}-1\end{array}$ & $\begin{array}{l}7.4472 \mathrm{E}-2 \\
6.9188 \mathrm{E}-1\end{array}$ & $\begin{array}{l}5.4744 \mathrm{E}-2 \\
4.9212 \mathrm{E}-1\end{array}$ & $\begin{array}{l}4.2041 E-2 \\
3.6557 E-1\end{array}$ & $\begin{array}{l}3.4260 \mathrm{E}-2 \\
2.8944 \mathrm{E}-1\end{array}$ \\
\hline 1.1 & $\begin{array}{l}1.2237 \mathrm{E}-1 \\
1.2317\end{array}$ & $\begin{array}{l}1.0596 \mathrm{E}-1 \\
1.0534\end{array}$ & $\begin{array}{l}9.3877 E-2 \\
9.2275 E-1\end{array}$ & $\begin{array}{l}8.5419 \mathrm{E}-2 \\
8.3167 \mathrm{E}-1\end{array}$ & $\begin{array}{l}8.1385 \mathrm{E}-2 \\
7.8836 \mathrm{E}-1\end{array}$ & $\begin{array}{l}7.7481 \mathrm{E}-2 \\
7.4653 \mathrm{E}-1\end{array}$ & $\begin{array}{l}7.4950 \mathrm{E}-2 \\
7.1948 \mathrm{E}-1\end{array}$ & $\begin{array}{l}5.2816 \mathrm{E}-2 \\
4.8516 \mathrm{E}-1\end{array}$ & $\begin{array}{l}3.9200 \mathrm{E}-2 \\
3.4438 \mathrm{E}-1\end{array}$ & $\begin{array}{l}3.1228 \mathrm{E}-2 \\
2.6412 \mathrm{E}-1\end{array}$ \\
\hline 1.2 & $\begin{array}{l}1.2907 \mathrm{E}-1 \\
1.3568\end{array}$ & $\begin{array}{l}1.0995 \mathrm{E}-1 \\
1.1382\end{array}$ & $\begin{array}{l}9.6025 \mathrm{E}-2 \\
9.7986 \mathrm{E}-1\end{array}$ & $\begin{array}{l}8.6373 E-2 \\
8.7072 E-1\end{array}$ & $\begin{array}{l}8.1803 E-2 \\
8.1924 E-1\end{array}$ & $\begin{array}{l}7.7403 \mathrm{E}-2 \\
7.6983 \mathrm{E}-1\end{array}$ & $\begin{array}{l}7.4564 \mathrm{E}-2 \\
7.3803 \mathrm{E}-1\end{array}$ & $\begin{array}{l}5.0319 \mathrm{E}-2 \\
4.7013 \mathrm{E}-1\end{array}$ & $\begin{array}{l}3.6162 \mathrm{E}-2 \\
3.1885 \mathrm{E}-1\end{array}$ & $\begin{array}{l}2.8263 \mathrm{E}-2 \\
2.3757 \mathrm{E}-1\end{array}$ \\
\hline 1.3 & $\begin{array}{l}1.3509 \mathrm{E}-1 \\
1.4832\end{array}$ & $\begin{array}{l}1.1314 \mathrm{E}-1 \\
1.2190\end{array}$ & $\begin{array}{l}9.7342 E-2 \\
1.0301\end{array}$ & $\begin{array}{l}8.6511 \mathrm{E}-2 \\
9.0155 \mathrm{E}-1\end{array}$ & $\begin{array}{l}8.1423 \mathrm{E}-2 \\
8.4146 \mathrm{E}-1\end{array}$ & $\begin{array}{l}7.6554 \mathrm{E}-2 \\
7.8418 \mathrm{E}-1\end{array}$ & $\begin{array}{l}7.3430 \mathrm{E}-2 \\
7.4755 \mathrm{E}-1\end{array}$ & $\begin{array}{l}4.7453 \mathrm{E}-2 \\
4.4860 \mathrm{E}-1\end{array}$ & $\begin{array}{l}3.3130 \mathrm{E}-2 \\
2.9120 \mathrm{E}-1\end{array}$ & $\begin{array}{l}2.5519 \mathrm{E}-2 \\
2.1179 \mathrm{E}-1\end{array}$ \\
\hline 1.4 & $\begin{array}{l}1.4043 \mathrm{E}-1 \\
1.6105\end{array}$ & $\begin{array}{l}1.1557 \mathrm{E}-1 \\
1.2952\end{array}$ & $\begin{array}{l}9.7890 \mathrm{E}-2 \\
1.0730\end{array}$ & $\begin{array}{l}8.5916 \mathrm{E}-2 \\
9.2388 \mathrm{E}-1\end{array}$ & $\begin{array}{l}8.0343 \mathrm{E}-2 \\
8.5493 \mathrm{E}-1\end{array}$ & $\begin{array}{l}7.5045 \mathrm{E}-2 \\
7.8970 \mathrm{E}-1\end{array}$ & $\begin{array}{l}7.1666 \mathrm{E}-2 \\
7.4829 \mathrm{E}-1\end{array}$ & $\begin{array}{l}4.4397 \mathrm{E}-2 \\
4.2232 \mathrm{E}-1\end{array}$ & $\begin{array}{l}3.0248 \mathrm{E}-2 \\
2.6337 \mathrm{E}-1\end{array}$ & $\begin{array}{l}2.3075 \mathrm{E}-2 \\
1.8812 \mathrm{E}-1\end{array}$ \\
\hline 1.45 & $\begin{array}{l}1.4286 \mathrm{E}-1 \\
1.6743\end{array}$ & $\begin{array}{l}1.1651 \mathrm{E}-1 \\
1.3314\end{array}$ & $\begin{array}{l}9.7897 \mathrm{E}-2 \\
1.0916\end{array}$ & $\begin{array}{l}8.5373 E-2 \\
9.3182 E-1\end{array}$ & $\begin{array}{l}7.9573 E-2 \\
8.5839 E-1\end{array}$ & $\begin{array}{l}7.4079 \mathrm{E}-2 \\
7.8924 \mathrm{E}-1\end{array}$ & $\begin{array}{l}7.0586 \mathrm{E}-2 \\
7.4552 \mathrm{E}-1\end{array}$ & $\begin{array}{l}4.2847 \mathrm{E}-2 \\
4.0795 \mathrm{E}-1\end{array}$ & $\begin{array}{l}2.8894 \mathrm{E}-2 \\
2.4986 \mathrm{E}-1\end{array}$ & $\begin{array}{l}2.1975 \mathrm{E}-2 \\
1.7732 \mathrm{E}-1\end{array}$ \\
\hline 1.6 & $\begin{array}{l}1.4919 \mathrm{E}-1 \\
1.8660\end{array}$ & $\begin{array}{l}1.1830 \mathrm{E}-1 \\
1.4314\end{array}$ & $\begin{array}{l}9.6948 \mathrm{~B}-2 \\
1.1351\end{array}$ & $\begin{array}{l}8.2892 E-2 \\
9.4277 E-1\end{array}$ & $\begin{array}{l}7.6490 \mathrm{E}-2 \\
8.5613 \mathrm{E}-1\end{array}$ & $\begin{array}{l}7.0500 \mathrm{E}-2 \\
7.7577 \mathrm{E}-1\end{array}$ & $\begin{array}{l}6.6733 \mathrm{E}-2 \\
7.2563 \mathrm{E}-1\end{array}$ & $\begin{array}{l}3.8291 \mathrm{E}-2 \\
3.6245 \mathrm{E}-1\end{array}$ & $\begin{array}{l}2.5252 \mathrm{E}-2 \\
2.1248 \mathrm{E}-1\end{array}$ & $\begin{array}{l}1.9148 \mathrm{E}-2 \\
1.4946 \mathrm{E}-1\end{array}$ \\
\hline 1.7 & $\begin{array}{l}1.5263 \mathrm{E}-1 \\
1.9934\end{array}$ & $\begin{array}{l}1.1868 \mathrm{E}-1 \\
1.4903\end{array}$ & $\begin{array}{l}9.5998 \mathrm{E}-2 \\
1.1537\end{array}$ & $\begin{array}{l}8.0647 \mathrm{E}-2 \\
9.3961 \mathrm{E}-1\end{array}$ & $\begin{array}{l}7.3922 \mathrm{E}-2 \\
8.4467 \mathrm{E}-1\end{array}$ & $\begin{array}{l}6.7688 \mathrm{E}-2 \\
7.5764 \mathrm{E}-1\end{array}$ & $\begin{array}{l}6.3800 \mathrm{E}-2 \\
7.0390 \mathrm{E}-1\end{array}$ & $\begin{array}{l}3.5444 \mathrm{E}-2 \\
3.3193 \mathrm{E}-1\end{array}$ & $\begin{array}{l}2.3188 \mathrm{E}-2 \\
1.9088 \mathrm{E}-1\end{array}$ & $\begin{array}{l}1.7616 \mathrm{E}-2 \\
1.3453 \mathrm{E}-1\end{array}$ \\
\hline 1.8 & $\begin{array}{l}1.5547 \mathrm{E}-1 \\
2.1197\end{array}$ & $\begin{array}{l}1.1848 \mathrm{E}-1 \\
1.5421\end{array}$ & $\begin{array}{l}9.3759 \mathrm{E}-2 \\
1.1639\end{array}$ & $\begin{array}{l}7.8037 \mathrm{E}-2 \\
9.2858 \mathrm{E}-1\end{array}$ & $\begin{array}{l}7.1062 \mathrm{E}-2 \\
8.2608 \mathrm{E}-1\end{array}$ & $\begin{array}{l}6.4660 \mathrm{E}-2 \\
7.3329 \mathrm{E}-1\end{array}$ & $\begin{array}{l}6.0700 \mathrm{E}-2 \\
6.7665 \mathrm{E}-1\end{array}$ & $\begin{array}{l}3.2816 \mathrm{E}-2 \\
3.0261 \mathrm{E}-1\end{array}$ & $\begin{array}{l}2.1401 \mathrm{E}-2 \\
1.7217 \mathrm{E}-1\end{array}$ & $\begin{array}{l}1.6318 \mathrm{E}-2 \\
1.2214 \mathrm{E}-1\end{array}$ \\
\hline 1.9 & $\begin{array}{l}1.5774 E-1 \\
2.2446\end{array}$ & $\begin{array}{l}1.1773 \mathrm{Z}-1 \\
1.5865\end{array}$ & $\begin{array}{l}9.1505 \mathrm{E}-2 \\
1.1656\end{array}$ & $\begin{array}{l}7.5152 \mathrm{E}-2 \\
9.1032 \mathrm{E}-1\end{array}$ & $\begin{array}{l}6.8003 \mathrm{E}-2 \\
8.0122 \mathrm{E}-1\end{array}$ & $\begin{array}{l}6.1510 \mathrm{E}-2 \\
7.0384 \mathrm{~B}-1\end{array}$ & $\begin{array}{l}5.7530 \mathrm{E}-2 \\
6.4510 \mathrm{E}-1\end{array}$ & $\begin{array}{l}3.0433 \mathrm{E}-2 \\
2.7527 \mathrm{E}-1\end{array}$ & $\begin{array}{l}1.9861 \mathrm{E}-2 \\
1.5621 \mathrm{E}-1\end{array}$ & $\begin{array}{l}1.5213 \mathrm{E}-2 \\
1.1188 \mathrm{E}-1\end{array}$ \\
\hline 2.0 & $\begin{array}{l}1.5946 \mathrm{E}-1 \\
2.3672\end{array}$ & $\begin{array}{l}1.1649 \mathrm{E}-1 \\
1.6229\end{array}$ & $\begin{array}{l}8.8913 \mathrm{E}-2 \\
1.1592\end{array}$ & $\begin{array}{l}7.2079 \mathrm{E}-2 \\
8.8562 \mathrm{E}-1\end{array}$ & $\begin{array}{l}6.4835 \mathrm{E}-2 \\
7.7114 \mathrm{~B}-1\end{array}$ & $\begin{array}{l}5.8325 \mathrm{E}-2 \\
6.7044 \mathrm{E}-1\end{array}$ & $\begin{array}{l}5.4371 \mathrm{E}-2 \\
6.1047 \mathrm{E}-1\end{array}$ & $\begin{array}{l}2.8300 \mathrm{E}-2 \\
2.5038 \mathrm{E}-1\end{array}$ & $\begin{array}{l}1.8533 \mathrm{E}-2 \\
1.4273 \mathrm{E}-1\end{array}$ & $\begin{array}{l}1.4263 \mathrm{E}-2 \\
1.0335 \mathrm{E}-1\end{array}$ \\
\hline 2.1 & $\begin{array}{l}1.6064 \mathrm{E}-1 \\
2.4870\end{array}$ & $\begin{array}{l}1.1481 \mathrm{E}-1 \\
1.6511\end{array}$ & $\begin{array}{l}8.6054 \mathrm{~B}-2 \\
1.1451\end{array}$ & $\begin{array}{l}6.8896 \mathrm{E}-2 \\
8.5544 \mathrm{E}-1\end{array}$ & $\begin{array}{l}6.1632 \mathrm{E}-2 \\
7.3693 \mathrm{E}-1\end{array}$ & $\begin{array}{l}5.5174 \mathrm{E}-2 \\
6.3428 \mathrm{E}-1\end{array}$ & $\begin{array}{l}5.1287 \mathrm{E}-2 \\
5.7397 \mathrm{E}-1\end{array}$ & $\begin{array}{l}2.6407 \mathrm{E}-2 \\
2.2817 \mathrm{E}-1\end{array}$ & $\begin{array}{l}1.7384 \mathrm{E}-2 \\
1.3138 \mathrm{E}-1\end{array}$ & $\begin{array}{l}1.3438 \mathrm{E}-2 \\
9.6183 \mathrm{E}-2\end{array}$ \\
\hline 2.2 & $\begin{array}{l}1.6131 \mathrm{E}-1 \\
2.6033\end{array}$ & $\begin{array}{l}1.1274 \mathrm{E}-1 \\
1.6707\end{array}$ & $\begin{array}{l}8.3000 \mathrm{E}-2 \\
1.123 \mathrm{~B}\end{array}$ & $\begin{array}{l}6.5675 \mathrm{E}-2 \\
8.2078 \mathrm{E}-1\end{array}$ & $\begin{array}{l}5.8460 \mathrm{E}-2 \\
6.9970 \mathrm{E}-1\end{array}$ & $\begin{array}{l}5.2115 \mathrm{E}-2 \\
5.9651 \mathrm{E}-1\end{array}$ & $\begin{array}{l}4.8330 \mathrm{E}-2 \\
5.3668 \mathrm{E}-1\end{array}$ & $\begin{array}{l}2.4737 \mathrm{E}-2 \\
2.0864 \mathrm{E}-1\end{array}$ & $\begin{array}{l}1.6384 \mathrm{E}-2 \\
1.2180 \mathrm{E}-1\end{array}$ & $\begin{array}{l}1.2713 \mathrm{E}-2 \\
9.0083 \mathrm{~B}-2\end{array}$ \\
\hline 2.3 & $\begin{array}{l}1.6150 \mathrm{E}-1 \\
2.7153\end{array}$ & $\begin{array}{l}1.1034 \mathrm{E}-1 \\
1.6817\end{array}$ & $\begin{array}{l}7.9815 \mathrm{E}-2 \\
1.0961\end{array}$ & $\begin{array}{l}6.2474 \mathrm{E}-2 \\
7.8271 \mathrm{E}-1\end{array}$ & $\begin{array}{l}5.5371 \mathrm{E}-2 \\
6.6056 \mathrm{E}-1\end{array}$ & $\begin{array}{l}4.9190 \mathrm{E}-2 \\
5.5815 \mathrm{E}-1\end{array}$ & $\begin{array}{l}4.5533 \mathrm{E}-2 \\
4.99588 \mathrm{E}-1\end{array}$ & $\begin{array}{l}2.3264 \mathrm{~B}-2 \\
1.9166 \mathrm{E}-1\end{array}$ & $\begin{array}{l}1.5505 \mathrm{E}-2 \\
1.1368 \mathrm{E}-1\end{array}$ & $\begin{array}{l}1.2071 \mathrm{E}-2 \\
8.4820 \mathrm{~B}-2\end{array}$ \\
\hline 2.4 & $\begin{array}{l}1.6124 \mathrm{E}-1 \\
2.8223\end{array}$ & $\begin{array}{l}1.0765 \mathrm{E}-1 \\
1.6840\end{array}$ & $\begin{array}{l}7.6563 \mathrm{E}-2 \\
1.0628\end{array}$ & $\begin{array}{l}5.9344 \mathrm{E}-2 \\
7.4226 \mathrm{E}-1\end{array}$ & $\begin{array}{l}5.2406 \mathrm{E}-2 \\
6.2050 \mathrm{E}-1\end{array}$ & $\begin{array}{l}4.6429 \mathrm{E}-2 \\
5.2010 \mathrm{E}-1\end{array}$ & $\begin{array}{l}4.2920 \mathrm{E}-2 \\
4.6346 \mathrm{E}-1\end{array}$ & $\begin{array}{l}2.1963 \mathrm{E}-2 \\
1.7699 \mathrm{E}-1\end{array}$ & $\begin{array}{l}1.4727 \mathrm{E}-2 \\
1.0671 \mathrm{E}-1\end{array}$ & $\begin{array}{l}1.1497 \mathrm{E}-2 \\
8.0220 \mathrm{E}-2\end{array}$ \\
\hline 2.5 & $\begin{array}{l}1.6054 \mathrm{E}-1 \\
2.9236\end{array}$ & $\begin{array}{l}1.0474 \mathrm{E}-1 \\
1.6778\end{array}$ & $\begin{array}{l}7.3296 \mathrm{E}-2 \\
1.0247\end{array}$ & $\begin{array}{l}5.6323 E-2 \\
7.0044 E-1\end{array}$ & $\begin{array}{l}4.9594 \mathrm{E}-2 \\
5.8045 \mathrm{E}-1\end{array}$ & $\begin{array}{l}4.3850 \mathrm{E}-2 \\
4.8313 \mathrm{E}-1\end{array}$ & $\begin{array}{l}4.0502 \mathrm{E}-2 \\
4.2897 \mathrm{E}-1\end{array}$ & $\begin{array}{l}2.0812 \mathrm{E}-2 \\
1.6436 \mathrm{E}-1\end{array}$ & $\begin{array}{l}1.4032 \mathrm{E}-2 \\
1.0068 \mathrm{E}-1\end{array}$ & $\begin{array}{l}1.0978 \mathrm{R}-2 \\
7.6153 \mathrm{E}-2\end{array}$ \\
\hline 2.6 & $\begin{array}{l}1.5944 \mathrm{E}-1 \\
3.0183\end{array}$ & $\begin{array}{l}1.0164 \mathrm{E}-1 \\
1.6634\end{array}$ & $\begin{array}{l}7.0060 \mathrm{E}-2 \\
9.8282 \mathrm{E}-1\end{array}$ & $\begin{array}{l}5.3441 \mathrm{E}-2 \\
6.5817 \mathrm{E}-1\end{array}$ & $\begin{array}{l}4.6952 \mathrm{E}-2 \\
5.4118 \mathrm{E}-1\end{array}$ & $\begin{array}{l}4.1461 \mathrm{E}-2 \\
4.4783 \mathrm{E}-1\end{array}$ & $\begin{array}{l}3.8280 \mathrm{E}-2 \\
3.9656 \mathrm{E}-1\end{array}$ & $\begin{array}{l}1.9788 \mathrm{E}-2 \\
1.5348 \mathrm{E}-1\end{array}$ & $\begin{array}{l}1.3406 \mathrm{E}-2 \\
9.5398 \mathrm{E}-2\end{array}$ & $\begin{array}{l}1.0508 \mathrm{E}-2 \\
7.2519 \mathrm{E}-2\end{array}$ \\
\hline 2.7 & $\begin{array}{l}1.5797 \mathrm{E}-1 \\
3.1059\end{array}$ & $\begin{array}{l}9.8424 E-2 \\
1.6412\end{array}$ & $\begin{array}{l}6.6893 \mathrm{E}-2 \\
9.3811 \mathrm{E}-1\end{array}$ & $\begin{array}{l}5.0717 \mathrm{E}-2 \\
6.1624 \mathrm{E}-1\end{array}$ & $\begin{array}{l}4.4491 \mathrm{E}-2 \\
5.0331 \mathrm{E}-1\end{array}$ & $\begin{array}{l}3.9262 \mathrm{E}-2 \\
4.1463 \mathrm{E}-1\end{array}$ & $\begin{array}{l}3.6249 \mathrm{E}-2 \\
3.6652 \mathrm{E}-1\end{array}$ & $\begin{array}{l}1.8872 \mathrm{E}-2 \\
1.4408 \mathrm{E}-1\end{array}$ & $\begin{array}{l}1.2838 \mathrm{E}-2 \\
9.0720 \mathrm{E}-2\end{array}$ & $\begin{array}{l}1.0079 \mathrm{E}-2 \\
6.9245 \mathrm{E}-2\end{array}$ \\
\hline 2.8 & $\begin{array}{l}1.5616 \mathrm{E}-1 \\
3.1856\end{array}$ & $\begin{array}{l}9.5120 \mathrm{E}-2 \\
1.6117\end{array}$ & $\begin{array}{l}6.3826 \mathrm{E}-2 \\
8.9149 \mathrm{E}-1\end{array}$ & $\begin{array}{l}4.8163 \mathrm{E}-2 \\
5.7535 \mathrm{E}-1\end{array}$ & $\begin{array}{l}4.2213 \mathrm{E}-2 \\
4.6734 \mathrm{E}-1\end{array}$ & $\begin{array}{l}3.7249 \mathrm{E}-2 \\
3.8382 \mathrm{E}-1\end{array}$ & $\begin{array}{l}3.4402 \mathrm{E}-2 \\
3.3903 \mathrm{E}-1\end{array}$ & $\begin{array}{l}1.8047 \mathrm{E}-2 \\
1.3591 \mathrm{E}-1\end{array}$ & $\begin{array}{l}1.2321 \mathrm{E}-2 \\
8.6536 \mathrm{E}-2\end{array}$ & $\begin{array}{l}9.6844 E-3 \\
6.6275 E-2\end{array}$ \\
\hline 2.9 & $\begin{array}{l}1.5404 \mathrm{E}-1 \\
3.2566\end{array}$ & $\begin{array}{l}9.1774 \mathrm{E}-2 \\
1.5757\end{array}$ & $\begin{array}{l}6.0884 \mathrm{E}-2 \\
8.4382 \mathrm{E}-1\end{array}$ & $\begin{array}{l}4.5784 \mathrm{E}-2 \\
5.3606 \mathrm{~B}-1\end{array}$ & $\begin{array}{l}4.0116 \mathrm{E}-2 \\
4.3362 \mathrm{E}-1\end{array}$ & $\begin{array}{l}3.5413 \mathrm{E}-2 \\
3.5555 \mathrm{E}-1\end{array}$ & $\begin{array}{l}3.2724 \mathrm{E}-2 \\
3.1412 \mathrm{E}-1\end{array}$ & $\begin{array}{l}1.7299 \mathrm{E}-2 \\
1.2876 \mathrm{E}-1\end{array}$ & $\begin{array}{l}1.1846 \mathrm{E}-2 \\
8.2763 \mathrm{E}-2\end{array}$ & $\begin{array}{l}9.32118-3 \\
6.3564 \mathrm{E}-2\end{array}$ \\
\hline 3.0 & $\begin{array}{l}1.5164 \mathrm{E}-1 \\
3.3186\end{array}$ & $\begin{array}{l}8.8424 \mathrm{E}-2 \\
1.5337\end{array}$ & $\begin{array}{l}5.8082 \mathrm{E}-2 \\
7.9591 \mathrm{E}-1\end{array}$ & $\begin{array}{l}4.3579 \mathrm{E}-2 \\
4.9877 \mathrm{E}-1\end{array}$ & $\begin{array}{l}3.8191 \mathrm{E}-2 \\
4.0236 \mathrm{E}-1\end{array}$ & $\begin{array}{l}3.3740 \mathrm{E}-2 \\
3.2986 \mathrm{E}-1\end{array}$ & $\begin{array}{l}3.12018-2 \\
2.9173 \mathrm{E}-1\end{array}$ & $\begin{array}{l}1.6618 \mathrm{E}-2 \\
1.2244 \mathrm{E}-1\end{array}$ & $\begin{array}{l}1.1408 \mathrm{E}-2 \\
7.9336 \mathrm{E}-2\end{array}$ & $\begin{array}{l}8.9849 \mathrm{E}-3 \\
6.1076 \mathrm{R}-2\end{array}$ \\
\hline 3.1 & $\begin{array}{l}1.4899 \mathrm{E}-1 \\
3.3710\end{array}$ & $\begin{array}{l}8.5102 \mathrm{E}-2 \\
1.4865\end{array}$ & $\begin{array}{l}5.5432 \mathrm{E}-2 \\
7.4846 \mathrm{E}-1\end{array}$ & $\begin{array}{l}4.1544 \mathrm{E}-2 \\
4.6381 \mathrm{E}-1\end{array}$ & $\begin{array}{l}3.6430 \mathrm{E}-2 \\
3.7367 \mathrm{E}-1\end{array}$ & $\begin{array}{l}3.2218 \mathrm{E}-2 \\
3.0670 \mathrm{E}-1\end{array}$ & $\begin{array}{l}2.9818 \mathrm{E}-2 \\
2.7175 \mathrm{E}-1\end{array}$ & $\begin{array}{l}1.5994 \mathrm{E}-2 \\
1.1682 \mathrm{E}-1\end{array}$ & $\begin{array}{l}1.1004 \mathrm{E}-2 \\
7.6204 \mathrm{E}-2\end{array}$ & $\begin{array}{l}8.6726 \mathrm{E}-3 \\
5.8782 \mathrm{E}-2\end{array}$ \\
\hline 3.2 & $\begin{array}{l}1.4613 \mathrm{E}-1 \\
3.4133\end{array}$ & $\begin{array}{l}8.1837 \mathrm{E}-2 \\
1.4351\end{array}$ & $\begin{array}{l}5.2940 \mathrm{E}-2 \\
7.0209 \mathrm{E}-1\end{array}$ & $\begin{array}{l}3.9672 \mathrm{E}-2 \\
4.3135 \mathrm{E}-1\end{array}$ & $\begin{array}{l}3.4820 \mathrm{E}-2 \\
3.4756 \mathrm{E}-1\end{array}$ & $\begin{array}{l}3.0832 \mathrm{E}-2 \\
2.8596 \mathrm{E}-1\end{array}$ & $\begin{array}{l}2.8560 \mathrm{E}-2 \\
3.5399 \mathrm{E}-1\end{array}$ & $\begin{array}{l}E-2 \\
E-1\end{array}$ & $\begin{array}{l}1.0628 \mathrm{E}-2 \\
7.3326 \mathrm{E}-2\end{array}$ & $\begin{array}{l}8.3846 \mathrm{E}-3 \\
5.6669 \mathrm{E}-2\end{array}$ \\
\hline 3.3 & $\begin{array}{l}1.4309 E-1 \\
3.4452\end{array}$ & $\begin{array}{l}7.8651 E-2 \\
1.3802\end{array}$ & $\begin{array}{l}5.0608 \mathrm{E}-2 \\
6.5732 \mathrm{E}-1\end{array}$ & $\begin{array}{l}3.7951 \mathrm{E}-2 \\
4.0148 \mathrm{E}-1\end{array}$ & $\begin{array}{l}3.3348 \mathrm{E}-2 \\
3.2398 \mathrm{E}-1\end{array}$ & $\begin{array}{l}2.9568 \mathrm{E}-2 \\
2.6746 \mathrm{E}-1\end{array}$ & $\begin{array}{l}2.74 \\
2.38\end{array}$ & $\begin{array}{l}1.488 \\
1.072\end{array}$ & $\begin{array}{l}1.0278 \mathrm{E}-2 \\
7.0670 \mathrm{E}-2\end{array}$ & $\begin{array}{l}8.1142 \mathrm{E}-3 \\
5.4707 \mathrm{E}-2\end{array}$ \\
\hline 3.4 & $\begin{array}{l}1.3990 \mathrm{E}-\mathrm{i} \\
3.4666\end{array}$ & $\begin{array}{l}7.5566 \mathrm{E}-2 \\
1.3226\end{array}$ & $\begin{array}{l}4.8432 \mathrm{E}-2 \\
6.1454 \mathrm{E}-1\end{array}$ & $\begin{array}{l}3.6371 \mathrm{E}-2 \\
3.7421 \mathrm{E}-1\end{array}$ & $\begin{array}{l}3.2001 \mathrm{E}-2 \\
3.0278 \mathrm{E}-1\end{array}$ & $\begin{array}{l}2.84 \\
2.51\end{array}$ & 2.24 & $=-2$ & $\begin{array}{l}9.9510 \mathrm{E}-3 \\
6.8208 \mathrm{E}-2\end{array}$ & $\begin{array}{l}7.8611 \mathrm{E}-3 \\
5.2880 \mathrm{E}-2\end{array}$ \\
\hline 4.4 & $\begin{array}{l}1.0549 \mathrm{E}-1 \\
3.1270\end{array}$ & $\begin{array}{l}5.1796 \mathrm{E}-2 \\
7.5232 \mathrm{E}-1\end{array}$ & $\begin{array}{l}3.3686 \mathrm{E}-2 \\
3.2433 \mathrm{E}-1\end{array}$ & $\begin{array}{l}2.5949 \mathrm{E}-2 \\
2.1455 \mathrm{E}-1\end{array}$ & $\begin{array}{l}2.3097 \mathrm{E}-2 \\
1.8266 \mathrm{E}-1\end{array}$ & $\begin{array}{l}2.0713 \mathrm{E}-2 \\
1.5858 \mathrm{E}-1\end{array}$ & $\begin{array}{l}1.9331 \mathrm{E}-2 \\
1.4551 \mathrm{E}-1\end{array}$ & $\begin{array}{l}1.0862 \\
7.4984\end{array}$ & $\begin{array}{l}7.57218-3 \\
5.0787 \mathrm{E}-2\end{array}$ & $\begin{array}{l}6.00218-3 \\
3.97298-2\end{array}$ \\
\hline 4.5 & $\begin{array}{l}1.0224 \mathrm{E}-1 \\
3.0472\end{array}$ & $\begin{array}{l}5.0116 \mathrm{E}-2 \\
7.0617 \mathrm{E}-1\end{array}$ & $\begin{array}{l}3.2720 \mathrm{E}-2 \\
3.0772 \mathrm{E}-1\end{array}$ & $\begin{array}{l}2.5257 \mathrm{E}-2 \\
2.0612 \mathrm{E}-2\end{array}$ & $\begin{array}{l}2.2498 \mathrm{E}-2 \\
1.7620 \mathrm{E}-1\end{array}$ & $\begin{array}{l}2.0186 \mathrm{E}-2 \\
1.5341 \mathrm{E}-1\end{array}$ & $\begin{array}{l}1.8845 \mathrm{E}-2 \\
1.4096 \mathrm{E}-1\end{array}$ & $\begin{array}{l}1.0605 \\
7.3034\end{array}$ & $\begin{array}{l}7.3964 \mathrm{E}-3 \\
4.9532 \mathrm{E}-2\end{array}$ & $\begin{array}{l}5.8641 \mathrm{E}-3 \\
3.8870 \mathrm{E}-2\end{array}$ \\
\hline 4.6 & $\begin{array}{l}9.9095 \mathrm{E}-2 \\
2.9616\end{array}$ & $\begin{array}{l}4.8541 E-2 \\
6.6293 E-1\end{array}$ & $\begin{array}{l}3.1814 \mathrm{E}-2 \\
2.9277 \mathrm{E}-1\end{array}$ & $\begin{array}{l}2.4606 \mathrm{E}-2 \\
1.9846 \mathrm{E}-1\end{array}$ & $\begin{array}{l}2.1931 \mathrm{E}-2 \\
1.7028 \mathrm{E}-1\end{array}$ & $\begin{array}{l}1.9687 \mathrm{E}-2 \\
1.4863 \mathrm{E}-1\end{array}$ & $\begin{array}{l}1.8384 \mathrm{E}-2 \\
1.3673 \mathrm{E}-1\end{array}$ & $\begin{array}{l}1.0360 \mathrm{E}-2 \\
7.1186 \mathrm{E}-2\end{array}$ & $\begin{array}{l}7.2288 \mathrm{E}-3 \\
4.8340 \mathrm{E}-2\end{array}$ & $\begin{array}{l}5.7323 E-3 \\
3.7857 E-2\end{array}$ \\
\hline
\end{tabular}


Table I (Continued)

\begin{tabular}{|c|c|c|c|c|c|c|c|c|}
\hline $\begin{array}{l}\text { Primary } \\
\text { Age (AE) }\end{array}$ & $\begin{array}{c}3 \times 10^{10} \\
30.5\end{array}$ & 32.0 & 50.0 & 64.0 & 128 & 256 & 512 & 1000 \\
\hline 1.0 & $\begin{array}{l}2.8850 \mathrm{E}-2 \\
2.3748 \mathrm{E}-1\end{array}$ & $\begin{array}{l}2.6038 \mathrm{E}-2 \\
2.1088 \mathrm{E}-1\end{array}$ & $\begin{array}{l}9.2109 \mathrm{E}-3 \\
6.2930 \mathrm{E}-2\end{array}$ & $\begin{array}{l}5.2634 \mathrm{E}-3 \\
3.4739 \mathrm{E}-2\end{array}$ & $\begin{array}{l}1.2539 \mathrm{E}-3 \\
7.9911 \mathrm{E}-3\end{array}$ & $\begin{array}{l}3.1024 \mathrm{E}-4 \\
1.9628 \mathrm{E}-3\end{array}$ & $\begin{array}{l}7.7367 \mathrm{E}-5 \\
4.8860 \mathrm{E}-4\end{array}$ & $\begin{array}{l}2.0269 \mathrm{E}-5 \\
1.2795 \mathrm{E}-4\end{array}$ \\
\hline 1.1 & $\begin{array}{l}2.5893 E-2 \\
2.1183 E-1\end{array}$ & $\begin{array}{l}2.3195 \mathrm{E}-2 \\
1.8598 \mathrm{E}-1\end{array}$ & $\begin{array}{l}8.0829 \mathrm{E}-3 \\
5.4969 \mathrm{E}-2\end{array}$ & $\begin{array}{l}4.7284 E-3 \\
3.1033 E-2\end{array}$ & $\begin{array}{l}1.1373 \mathrm{E}-3 \\
7.2413 \mathrm{E}-3\end{array}$ & $\begin{array}{l}2.8189 \mathrm{E}-4 \\
1.7830 \mathrm{E}-3\end{array}$ & $\begin{array}{l}7.0324 \mathrm{E}-5 \\
4.4410 \mathrm{E}-4\end{array}$ & $\begin{array}{l}1.8426 \mathrm{E}-5 \\
1.1631 \mathrm{E}-4\end{array}$ \\
\hline 1.2 & $\begin{array}{l}2.3172 \mathrm{E}-2 \\
1.8710 \mathrm{E}-1\end{array}$ & $\begin{array}{l}2.0663 \mathrm{E}-2 \\
1.6299 \mathrm{E}-1\end{array}$ & $\begin{array}{l}7.2784 \mathrm{E}-3 \\
4.8982 \mathrm{E}-2\end{array}$ & $\begin{array}{l}4.2978 E-3 \\
2.8091 E-2\end{array}$ & $\begin{array}{l}1.0407 \mathrm{E}-3 \\
6.6213 \mathrm{E}-3\end{array}$ & $\begin{array}{l}2.5829 \mathrm{E}-4 \\
1.6334 \mathrm{E}-3\end{array}$ & $\begin{array}{l}6.4457 \mathrm{E}-5 \\
4.0703 \mathrm{E}-4\end{array}$ & $\begin{array}{l}1.6890 \mathrm{E}-5 \\
1.0662 \mathrm{E}-4\end{array}$ \\
\hline 1.3 & $\begin{array}{l}2.0781 \mathrm{E}-2 \\
1.6475 \mathrm{E}-1\end{array}$ & $\begin{array}{l}1.8495 \mathrm{E}-2 \\
1.4295 \mathrm{E}-1\end{array}$ & $\begin{array}{l}6.6340 \mathrm{E}-3 \\
4.4309 \mathrm{E}-2\end{array}$ & $\begin{array}{l}3.9419 \mathrm{E}-3 \\
2.5682 \mathrm{E}-2\end{array}$ & $\begin{array}{l}9.5934 \mathrm{E}-4 \\
6.0999 \mathrm{E}-3\end{array}$ & $\begin{array}{l}2.3834 E-4 \\
1.5071 E-3\end{array}$ & $\begin{array}{l}5.9494 \mathrm{E}-5 \\
3.7568 \mathrm{E}-4\end{array}$ & $\begin{array}{l}1.5590 \mathrm{E}-5 \\
9.8411 \mathrm{E}-5\end{array}$ \\
\hline 1.4 & $\begin{array}{l}1.8740 \mathrm{E}-2 \\
1.4544 \mathrm{E}-1\end{array}$ & $\begin{array}{l}1.6683 \mathrm{E}-2 \\
1.2616 \mathrm{E}-1\end{array}$ & $\begin{array}{l}6.1031 \mathrm{E}-3 \\
4.0532 \mathrm{E}-2\end{array}$ & $\begin{array}{l}3.6429 E-3 \\
2.3670 E-2\end{array}$ & $\begin{array}{l}8.8987 \mathrm{E}-4 \\
5.6550 \mathrm{E}-3\end{array}$ & $\begin{array}{l}2.2126 \mathrm{E}-4 \\
1.3989 \mathrm{E}-3\end{array}$ & $\begin{array}{l}5.5241 E-5 \\
3.4881 E-4\end{array}$ & $\begin{array}{l}1.4476 \mathrm{E}-5 \\
9.1380 \mathrm{E}-5\end{array}$ \\
\hline 1.45 & $\begin{array}{l}1.7846 \mathrm{E}-2 \\
1.3698 \mathrm{E}-1\end{array}$ & $\begin{array}{l}1.5897 \mathrm{E}-2 \\
1.1894 \mathrm{E}-1\end{array}$ & $\begin{array}{l}5.8703 \mathrm{E}-3 \\
3.8896 \mathrm{E}-2\end{array}$ & $\begin{array}{l}3.5102 E-3 \\
2.2782 E-2\end{array}$ & $\begin{array}{l}8.5880 \mathrm{E}-4 \\
5.4562 \mathrm{E}-3\end{array}$ & $\begin{array}{l}2.1361 \mathrm{E}-4 \\
1.3504 \mathrm{E}-3\end{array}$ & $\begin{array}{l}5.3334 \mathrm{E}-5 \\
3.3677 \mathrm{E}-4\end{array}$ & $\begin{array}{l}1.3977 \mathrm{E}-5 \\
8.8228 \mathrm{E}-5\end{array}$ \\
\hline 1.6 & $\begin{array}{l}1.5599 \mathrm{E}-3 \\
1.1600 \mathrm{E}-1\end{array}$ & $\begin{array}{l}1.3939 \mathrm{E}-2 \\
1.0130 \mathrm{E}-1\end{array}$ & $\begin{array}{l}5.2722 \mathrm{E}-3 \\
3.4735 \mathrm{E}-2\end{array}$ & $\begin{array}{l}3.1656 E-3 \\
2.0485 E-2\end{array}$ & $\begin{array}{l}7.7741 \mathrm{E}-4 \\
4.9360 \mathrm{E}-3\end{array}$ & $\begin{array}{l}1.9353 \mathrm{E}-4 \\
1.2233 \mathrm{E}-3\end{array}$ & $\begin{array}{l}4.8331 \mathrm{E}-5 \\
3.0516 \mathrm{E}-4\end{array}$ & $\begin{array}{l}1.2666 \mathrm{E}-5 \\
7.9954 \mathrm{E}-5\end{array}$ \\
\hline 1.7 & $\begin{array}{l}1.4404 \mathrm{E}-2 \\
1.0515 \mathrm{E}-1\end{array}$ & $\begin{array}{l}1.2900 \mathrm{E}-2 \\
9.2282 \mathrm{E}-2\end{array}$ & $\begin{array}{l}4.9390 \mathrm{E}-3 \\
3.2442 \mathrm{E}-2\end{array}$ & $\begin{array}{l}2.9718 \mathrm{E}-3 \\
1.9200 \mathrm{E}-2\end{array}$ & $\begin{array}{l}7.3125 \mathrm{E}-4 \\
4.6413 \mathrm{E}-3\end{array}$ & $\begin{array}{l}1.8212 \mathrm{E}-4 \\
1.1511 \mathrm{E}-3\end{array}$ & $\begin{array}{l}4.5486 \mathrm{E}-5 \\
2.8720 \mathrm{E}-4\end{array}$ & $\begin{array}{l}1.1921 \mathrm{E}-5 \\
7.5250 \mathrm{E}-5\end{array}$ \\
\hline 1.8 & $\begin{array}{l}1.3394 \mathrm{E}-2 \\
9.6277 \mathrm{E}-2\end{array}$ & $\begin{array}{l}1.2022 \mathrm{E}-2 \\
8.4895 \mathrm{E}-2\end{array}$ & $\begin{array}{l}4.6479 \mathrm{E}-3 \\
3.0449 \mathrm{E}-2\end{array}$ & $\begin{array}{l}2.8007 E-3 \\
1.8069 E-2\end{array}$ & $\begin{array}{l}6.9028 \mathrm{E}-4 \\
4.3799 \mathrm{E}-3\end{array}$ & $\begin{array}{l}1.7198 \mathrm{E}-4 \\
1.0869 \mathrm{E}-3\end{array}$ & $\begin{array}{l}4.2958 E-5 \\
2.7123 E-4\end{array}$ & $\begin{array}{l}1.1259 \mathrm{E}-5 \\
7.1068 \mathrm{E}-5\end{array}$ \\
\hline 1.9 & $\begin{array}{l}1.2533 \mathrm{E}-2 \\
8.8941 \mathrm{E}-2\end{array}$ & $\begin{array}{l}1.1269 \mathrm{E}-2 \\
7.8753 \mathrm{E}-2\end{array}$ & $\begin{array}{l}4.3897 E-3 \\
2.8692 E-2\end{array}$ & $\begin{array}{l}2.6485 \mathrm{E}-3 \\
1.7066 \mathrm{E}-2\end{array}$ & $\begin{array}{l}6.5367 \mathrm{E}-4 \\
4.1464 \mathrm{E}-3\end{array}$ & $\begin{array}{l}1.6291 \mathrm{E}-4 \\
1.0295 \mathrm{E}-3\end{array}$ & $\begin{array}{l}4.0696 \mathrm{E}-5 \\
2.5694 \mathrm{E}-4\end{array}$ & $\begin{array}{l}1.0666 \mathrm{E}-5 \\
6.7327 \mathrm{E}-5\end{array}$ \\
\hline 2.0 & $\begin{array}{l}1.1787 \mathrm{E}-2 \\
8.2790 \mathrm{E}-2\end{array}$ & $\begin{array}{l}1.0614 \mathrm{E}-2 \\
7.3558 \mathrm{E}-2\end{array}$ & $\begin{array}{l}4.1592 \mathrm{E}-3 \\
2.7132 \mathrm{E}-2\end{array}$ & $\begin{array}{l}2.5123 \mathrm{E}-3 \\
1.6170 \mathrm{E}-2\end{array}$ & $\begin{array}{l}6.2076 \mathrm{E}-4 \\
3.9367 \mathrm{E}-3\end{array}$ & $\begin{array}{l}1.5475 \mathrm{E}-4 \\
9.7789 \mathrm{E}-4\end{array}$ & $\begin{array}{l}3.8660 \mathrm{E}-5 \\
2.4408 \mathrm{E}-4\end{array}$ & $\begin{array}{l}1.0133 \mathrm{E}-5 \\
6.3960 \mathrm{E}-5\end{array}$ \\
\hline 2.1 & $\begin{array}{l}1.1135 \mathrm{E}-2 \\
7.7551 \mathrm{E}-2\end{array}$ & $\begin{array}{l}1.0037 \mathrm{E}-2 \\
6.9092 \mathrm{E}-2\end{array}$ & $\begin{array}{l}3.9523 \mathrm{E}-3 \\
2.5737 \mathrm{E}-2\end{array}$ & $\begin{array}{l}2.3895 \mathrm{E}-3 \\
1.5364 \mathrm{E}-2\end{array}$ & $\begin{array}{l}5.9102 \mathrm{E}-4 \\
3.7472 \mathrm{E}-3\end{array}$ & $\begin{array}{l}1.4737 \mathrm{E}-4 \\
9.3120 \mathrm{E}-4\end{array}$ & $\begin{array}{l}3.6819 \mathrm{E}-5 \\
2.3245 \mathrm{E}-4\end{array}$ & $\begin{array}{l}9.6503 E-6 \\
6.0914 E-5\end{array}$ \\
\hline 2.2 & $\begin{array}{l}1.0557 \mathrm{E}-2 \\
7.3021 \mathrm{E}-2\end{array}$ & $\begin{array}{l}9.5252 \mathrm{E}-3 \\
6.5195 \mathrm{E}-2\end{array}$ & $\begin{array}{l}3.7653 \mathrm{E}-3 \\
2.4481 \mathrm{E}-2\end{array}$ & $\begin{array}{l}2.2783 \mathrm{E}-3 \\
1.4636 \mathrm{E}-2\end{array}$ & $\begin{array}{l}5.6400 \mathrm{E}-4 \\
3.5751 \mathrm{E}-3\end{array}$ & $\begin{array}{l}1.4066 \mathrm{E}-4 \\
8.8876 \mathrm{E}-4\end{array}$ & $\begin{array}{l}3.5145 \mathrm{E}-5 \\
2.2188 \mathrm{E}-4\end{array}$ & $\begin{array}{l}9.2116 \mathrm{E}-6 \\
5.8145 \mathrm{E}-5\end{array}$ \\
\hline 2.3 & $\begin{array}{l}1.0041 \mathrm{E}-2 \\
6.9049 \mathrm{E}-2\end{array}$ & $\begin{array}{l}9.0660 \mathrm{E}-3 \\
6.1753 \mathrm{E}-2\end{array}$ & $\begin{array}{l}3.5955 \mathrm{E}-3 \\
2.3344 \mathrm{E}-2\end{array}$ & $\begin{array}{l}2.1770 \mathrm{E}-3 \\
1.3975 \mathrm{E}-2\end{array}$ & $\begin{array}{l}5.3935 E-4 \\
3.4182 \mathrm{E}-3\end{array}$ & $\begin{array}{l}1.3454 E-4 \\
8.5003 E-4\end{array}$ & $\begin{array}{l}3.3616 \mathrm{E}-5 \\
2.1223 \mathrm{E}-4\end{array}$ & $\begin{array}{l}8.8110 \mathrm{E}-6 \\
5.5616 \mathrm{E}-5\end{array}$ \\
\hline 2.4 & $\begin{array}{l}9.5758 \mathrm{E}-3 \\
6.5528 \mathrm{E}-2\end{array}$ & $\begin{array}{l}8.6513 \mathrm{E}-3 \\
5.8684 \mathrm{E}-2\end{array}$ & $\begin{array}{l}3.4405 E-3 \\
2.2309 \mathrm{E}-2\end{array}$ & $\begin{array}{l}2.0845 \mathrm{E}-3 \\
1.3371 \mathrm{E}-2\end{array}$ & $\begin{array}{l}5.1677 \mathrm{E}-4 \\
3.2745 \mathrm{E}-3\end{array}$ & $\begin{array}{l}1.2893 \mathrm{E}-4 \\
8.1454 \mathrm{E}-4\end{array}$ & $\begin{array}{l}3.2215 \mathrm{E}-5 \\
2.0338 \mathrm{E}-4\end{array}$ & $\begin{array}{l}8.4439 E-6 \\
5.3299 E-5\end{array}$ \\
\hline 2.5 & $\begin{array}{l}9.1545 \mathrm{E}-3 \\
6.2376 \mathrm{E}-2\end{array}$ & $\begin{array}{l}8.2745 E-3 \\
5.5923 E-2\end{array}$ & $\begin{array}{l}3.2986 \mathrm{E}-3 \\
2.1364 \mathrm{E}-2\end{array}$ & $\begin{array}{l}1.9996 \mathrm{E}-3 \\
1.2817 \mathrm{E}-2\end{array}$ & $\begin{array}{l}4.9600 \mathrm{E}-4 \\
3.1425 \mathrm{E}-3\end{array}$ & $\begin{array}{l}1.2376 \mathrm{E}-4 \\
7.8189 \mathrm{E}-4\end{array}$ & $\begin{array}{l}3.0926 \mathrm{E}-5 \\
1.9524 \mathrm{E}-4\end{array}$ & $\begin{array}{l}8.1061 E-6 \\
5.1166 E-5\end{array}$ \\
\hline 2.6 & $\begin{array}{l}8.7702 \mathrm{E}-3 \\
5.9533 \mathrm{E}-2\end{array}$ & $\begin{array}{l}7.9302 \mathrm{E}-3 \\
5.3422 \mathrm{E}-2\end{array}$ & $\begin{array}{l}3.1680 \mathrm{E}-3 \\
2.0496 \mathrm{E}-2\end{array}$ & $\begin{array}{l}1.9213 \mathrm{E}-3 \\
1.2308 \mathrm{E}-2\end{array}$ & $\begin{array}{l}4.7685 \mathrm{E}-4 \\
3.0207 \mathrm{E}-3\end{array}$ & $\begin{array}{l}1.1900 \mathrm{E}-4 \\
7.5176 \mathrm{E}-4\end{array}$ & $\begin{array}{l}2.9736 \mathrm{E}-5 \\
1.8773 \mathrm{E}-4\end{array}$ & $\begin{array}{l}7.7943 \mathrm{E}-6 \\
4.9198 \mathrm{E}-5\end{array}$ \\
\hline 2.7 & $\begin{array}{l}8.4181 \mathrm{E}-3 \\
5.6951 \mathrm{E}-2\end{array}$ & $\begin{array}{l}7.6140 \mathrm{E}-3 \\
5.1145 \mathrm{E}-2\end{array}$ & $\begin{array}{l}3.0475 \mathrm{E}-3 \\
1.9697 \mathrm{E}-2\end{array}$ & $\begin{array}{l}1.8490 \mathrm{E}-3 \\
1.1838 \mathrm{E}-2\end{array}$ & $\begin{array}{l}4.5912 \mathrm{E}-4 \\
2.9079 \mathrm{E}-3\end{array}$ & $\begin{array}{l}1.1459 \mathrm{E}-4 \\
7.2386 \mathrm{E}-4\end{array}$ & $\begin{array}{l}2.8635 E-5 \\
1.8077 E-4\end{array}$ & $\begin{array}{l}7.5056 \mathrm{E}-6 \\
4.7376 \mathrm{E}-5\end{array}$ \\
\hline 2.8 & $\begin{array}{l}8.0938 \mathrm{E}-3 \\
5.4593 \mathrm{E}-2\end{array}$ & $\begin{array}{l}7.3252 \mathrm{E}-3 \\
4.9067 \mathrm{E}-2\end{array}$ & $\begin{array}{l}2.9360 \mathrm{E}-3 \\
1.8959 \mathrm{E}-2\end{array}$ & $\begin{array}{l}1.7820 \mathrm{E}-3 \\
1.1403 \mathrm{E}-2\end{array}$ & $\begin{array}{l}4.4266 \mathrm{E}-4 \\
2.8034 \mathrm{E}-3\end{array}$ & $\begin{array}{l}1.1049 \mathrm{E}-4 \\
6.9797 \mathrm{E}-4\end{array}$ & $\begin{array}{l}2.7612 \mathrm{E}-5 \\
1.7431 \mathrm{E}-4\end{array}$ & $\begin{array}{l}7.2375 E-6 \\
4.5684 E-5\end{array}$ \\
\hline 2.9 & $\begin{array}{l}7.7939 \mathrm{E}-3 \\
5.2428 \mathrm{E}-2\end{array}$ & $\begin{array}{l}7.0569 \mathrm{E}-3 \\
4.7156 \mathrm{E}-2\end{array}$ & $\begin{array}{l}2.8324 \mathrm{E}-3 \\
1.8275 \mathrm{E}-2\end{array}$ & $\begin{array}{l}1.7197 \mathrm{E}-3 \\
1.0999 \mathrm{E}-2\end{array}$ & $\begin{array}{l}4.2734 \mathrm{E}-4 \\
2.7060 \mathrm{E}-3\end{array}$ & $\begin{array}{l}1.0668 \mathrm{E}-4 \\
6.7386 \mathrm{E}-4\end{array}$ & $\begin{array}{l}2.6659 \mathrm{E}-5 \\
1.6830 \mathrm{E}-4\end{array}$ & $\begin{array}{l}6.9880 \mathrm{E}-6 \\
4.4108 \mathrm{E}-5\end{array}$ \\
\hline 3.0 & $\begin{array}{l}7.5196 \mathrm{E}-3 \\
5.0447 \mathrm{E}-2\end{array}$ & $\begin{array}{l}6.8080 \mathrm{E}-3 \\
4.5393 \mathrm{E}-2\end{array}$ & $\begin{array}{l}2.7359 E-3 \\
1.7639 E-2\end{array}$ & $\begin{array}{l}1.6616 \mathrm{E}-3 \\
1.0623 \mathrm{E}-2\end{array}$ & $\begin{array}{l}4.1305 E-4 \\
2.6153 E-3\end{array}$ & $\begin{array}{l}1.0312 E-4 \\
6.5136 E-4\end{array}$ & $\begin{array}{l}2.5771 \mathrm{E}-5 \\
1.6269 \mathrm{E}-4\end{array}$ & $\begin{array}{l}6.7550 \mathrm{E}-6 \\
4.2638 \mathrm{E}-5\end{array}$ \\
\hline 3.1 & $\begin{array}{l}7.2624 \mathrm{E}-3 \\
4.8609 \mathrm{E}-2\end{array}$ & $\begin{array}{l}6.5765 \mathrm{E}-3 \\
4.3760 \mathrm{E}-2\end{array}$ & $\begin{array}{l}2.6458 \mathrm{E}-3 \\
1.7046 \mathrm{E}-2\end{array}$ & $\begin{array}{l}1.6074 E-3 \\
1.0271 E-2\end{array}$ & $\begin{array}{l}3.9969 \mathrm{E}-4 \\
2.5304 \mathrm{E}-3\end{array}$ & $\begin{array}{l}9.9789 \mathrm{E}-5 \\
6.3032 \mathrm{E}-4\end{array}$ & $\begin{array}{l}2.4939 \mathrm{E}-5 \\
1.5744 \mathrm{E}-4\end{array}$ & $\begin{array}{l}6.5371 \mathrm{E}-6 \\
4.1262 \mathrm{E}-5\end{array}$ \\
\hline 3.2 & $\begin{array}{l}7.0226 \mathrm{E}-3 \\
4.6905 \mathrm{E}-2\end{array}$ & $\begin{array}{l}6.3606 \mathrm{E}-3 \\
4.2243 \mathrm{E}-2\end{array}$ & $\begin{array}{l}2.5615 \mathrm{E}-3 \\
1.6491 \mathrm{E}-2\end{array}$ & $\begin{array}{l}1.5566 \mathrm{E}-3 \\
9.9427 \mathrm{E}-3\end{array}$ & $\begin{array}{l}3.8716 \mathrm{E}-4 \\
2.4509 \mathrm{E}-3\end{array}$ & $\begin{array}{l}9.6669 \mathrm{E}-5 \\
6.1059 \mathrm{E}-4\end{array}$ & $\begin{array}{l}2.4160 \mathrm{E}-5 \\
1.5252 \mathrm{E}-4\end{array}$ & $\begin{array}{l}6.3328 \mathrm{E}-6 \\
3.9973 \mathrm{E}-5\end{array}$ \\
\hline 3.3 & $\begin{array}{l}6.7986 \mathrm{E}-3 \\
4.5318 \mathrm{E}-2\end{array}$ & $\begin{array}{l}6.1587 \mathrm{E}-3 \\
4.0831 \mathrm{E}-2\end{array}$ & $\begin{array}{l}2.4825 \mathrm{E}-3 \\
1.5973 \mathrm{E}-2\end{array}$ & $\begin{array}{l}1.5089 E-3 \\
9.6345 E-3\end{array}$ & $\begin{array}{l}3.7540 \mathrm{E}-4 \\
2.3762 \mathrm{E}-3\end{array}$ & $\begin{array}{l}9.3737 \mathrm{E}-5 \\
5.9206 \mathrm{E}-4\end{array}$ & $\begin{array}{l}2.3427 \mathrm{E}-5 \\
1.4789 \mathrm{E}-4\end{array}$ & $\begin{array}{l}6.1409 \mathrm{E}-6 \\
3.8761 \mathrm{E}-5\end{array}$ \\
\hline 3.4 & $\begin{array}{l}6.5886 \mathrm{E}-3 \\
4.3839 \mathrm{E}-2\end{array}$ & $\begin{array}{l}5.9694 \mathrm{E}-3 \\
3.9512 \mathrm{E}-2\end{array}$ & $\begin{array}{l}2.4082 \mathrm{E}-3 \\
1.5486 \mathrm{E}-2\end{array}$ & $\begin{array}{l}1.4640 \mathrm{E}-3 \\
9.3449 \mathrm{E}-3\end{array}$ & $\begin{array}{l}3.6433 \mathrm{E}-4 \\
2.3059 \mathrm{E}-3\end{array}$ & $\begin{array}{l}9.0979 \mathrm{E}-5 \\
5.7463 \mathrm{E}-4\end{array}$ & $\begin{array}{l}2.2738 \mathrm{E}-5 \\
1.4354 \mathrm{E}-4\end{array}$ & $\begin{array}{l}5.9603 \mathrm{E}-6 \\
3.7621 \mathrm{E}-5\end{array}$ \\
\hline 4.4 & $\begin{array}{l}5.0409 \mathrm{E}-3 \\
3.3103 \mathrm{E}-2\end{array}$ & $\begin{array}{l}4.5716 \mathrm{E}-3 \\
2.9907 \mathrm{E}-2\end{array}$ & $\begin{array}{l}1.8544 \mathrm{E}-3 \\
1.1872 \mathrm{E}-2\end{array}$ & $\begin{array}{l}1.1289 \mathrm{E}-3 \\
7.1871 \mathrm{E}-3\end{array}$ & $\begin{array}{l}2.8138 \mathrm{~B}-4 \\
1.7798 \mathrm{E}-3\end{array}$ & $\begin{array}{l}7.0293 \mathrm{E}-5 \\
4.4390 \mathrm{E}-4\end{array}$ & $\begin{array}{l}1.7570 \mathrm{E}-5 \\
1.1091 \mathrm{E}-4\end{array}$ & $\begin{array}{l}4.6056 \mathrm{E}-6 \\
2.9070 \mathrm{E}-5\end{array}$ \\
\hline 4.5 & $\begin{array}{l}4.9256 \mathrm{E}-3 \\
3.2316 \mathrm{E}-2\end{array}$ & $\begin{array}{l}4.4674 \mathrm{E}-3 \\
2.9201 \mathrm{E}-2\end{array}$ & $\begin{array}{l}1.8128 \mathrm{E}-3 \\
1.1602 \mathrm{E}-2\end{array}$ & $\begin{array}{l}1.1037 \mathrm{E}-3 \\
7.0250 \mathrm{E}-3\end{array}$ & $\begin{array}{l}2.7512 \mathrm{E}-4 \\
1.7401 \mathrm{E}-3\end{array}$ & $\begin{array}{l}6.8730 \mathrm{E}-5 \\
4.3403 \mathrm{E}-4\end{array}$ & $\begin{array}{l}1.7179 \mathrm{E}-5 \\
1.0845 \mathrm{E}-4\end{array}$ & $\begin{array}{l}4.5033 E-6 \\
2.8424 E-5\end{array}$ \\
\hline 4.6 & $\begin{array}{l}4.8156 \mathrm{E}-3 \\
3.1565 \mathrm{E}-2\end{array}$ & $\begin{array}{l}4.3678 E-3 \\
2.8527 E-2\end{array}$ & $\begin{array}{l}1.7730 \mathrm{E}-3 \\
1.1344 \mathrm{E}-2\end{array}$ & $\begin{array}{l}1.0795 \mathrm{E}-3 \\
6.8701 \mathrm{E}-3\end{array}$ & $\begin{array}{l}2.6913 \mathrm{E}-4 \\
1.7021 \mathrm{E}-3\end{array}$ & $\begin{array}{l}6.7235 \mathrm{E}-5 \\
4.2458 \mathrm{E}-4\end{array}$ & $\begin{array}{l}1.6806 \mathrm{E}-5 \\
1.0609 \mathrm{E}-4\end{array}$ & $\begin{array}{l}4.4054 \mathrm{E}-6 \\
2.7806 \mathrm{E}-5\end{array}$ \\
\hline
\end{tabular}


TABLE $2 a$

$$
\begin{aligned}
& \frac{d r_{\lambda}}{d r_{\lambda^{\prime}}}=\frac{e^{\lambda t} \lambda}{e^{\lambda^{\prime} t} \lambda^{\prime}} \frac{\sum_{l=0}^{\infty} \frac{(-1)^{l} q_{l}(\lambda t)^{l}}{l !(l+2)}}{\sum_{l=0}^{\infty} \frac{(-1)^{l} q_{l}\left(\lambda^{\prime} t\right)^{l}}{l !(l+2)}} \\
& \text { where } \quad q_{l}=\int_{0}^{\pi / 2} \cos ^{l} \theta d \theta
\end{aligned}
$$

Asymptotic Behavior of Linearly Time-Dependent

\begin{tabular}{|c|c|c|}
\hline$t=\underset{\left(10^{9} \text { years }\right)}{\text { Primary Age }}$ & $d r_{\lambda} / d r_{\lambda^{\prime}} *$ & $\begin{array}{c}\text { Intercept on } \\
r_{\lambda^{\prime}}=\mathrm{Pb}^{206} / \mathrm{U}^{238} \\
\text { Axis of Asymptotic } \\
\text { Diffusion Curve }\end{array}$ \\
\hline 1.0 & 10.429 & 0.00893 \\
\hline 1.1 & 11.002 & 0.01071 \\
\hline 1.2 & 11.614 & 0.01266 \\
\hline 1.3 & 12.267 & 0.01474 \\
\hline 1.4 & 12.964 & 0.01697 \\
\hline 1.5 & 13.708 & 0.01932 \\
\hline 1.6 & 14.502 & 0.02180 \\
\hline 1.7 & 15.351 & 0.02440 \\
\hline 1.8 & 16.258 & 0.02712 \\
\hline 1.9 & 17.228 & 0.02998 \\
\hline 2.0 & 18.264 & 0.03292 \\
\hline 2.1 & 19.373 & 0.03599 \\
\hline 2.2 & 20.559 & 0.03914 \\
\hline 2.3 & 21.828 & 0.04241 \\
\hline 2.4 & 23.186 & 0.04576 \\
\hline 2.5 & 24.641 & 0.04922 \\
\hline 2.6 & 26.198 & 0.05275 \\
\hline 2.7 & 27.866 & 0.05637 \\
\hline 2.8 & 29.653 & 0.06007 \\
\hline 2.9 & 31.568 & 0.06385 \\
\hline 3.0 & 33.621 & 0.06772 \\
\hline 3.1 & 36.069 & \\
\hline 3.2 & 38.182 & \\
\hline 3.3 & 40.714 & \\
\hline 3.4 & 43.430 & \\
\hline 3.8 & 56.434 & \\
\hline 4.0 & 64.464 & \\
\hline 4.25 & 76.265 & \\
\hline 4.5 & 90.397 & \\
\hline
\end{tabular}
(Radiation Damage) Diffusion Curves

*These values of $d r_{\lambda} / d r_{\lambda^{\prime}}$ are correct to as many figures as shown.

would be calculated for the $D_{0}$ model. Figure 3 shows a comparison of the two models $D=D_{0}$ and $D=D_{1} \tau$ for the case of spherical geometry. The lines $A$ and $B$ represent the extrapolated asymptotic slopes at the intersection with concordia, and $A^{\prime}$ and $B^{\prime}$ are the curves for the $D_{0}$ and $D_{1} \tau$ models, respectively. From comparison of the separation between $A^{\prime}$ and $A$ and
$B^{\prime}$ and $B$ it is clear that the irradiation model is closer to being linear over a wider region. $A$ b careful inspection of all the various continuous diffusion models shows them to have a con. siderable curvature even in the so-called 'linear' .

TABLE $2 b$

$$
\frac{d r_{\lambda}}{d r_{\lambda^{\prime}}}=\frac{e^{\lambda t} \lambda}{e^{\lambda^{\prime} t} \lambda^{\prime}}\left[\frac{\frac{t^{1 / 2}}{\lambda}-\frac{e^{-\lambda t}}{\lambda^{3 / 2}} \int_{0}^{\sqrt{\lambda t}} e^{x^{2}} d x}{\frac{t^{1 / 2}}{\lambda^{\prime}}-\frac{e^{-\lambda^{\prime} t}}{\left(\lambda^{\prime}\right)^{3 / 2}} \int_{0}^{\sqrt{\lambda^{\prime} t}} e^{x^{2}} d x}\right]
$$

\begin{tabular}{|c|c|c|}
\hline$t=\underset{\left(10^{9} \text { years }\right)}{\text { Primary Age }}$ & $d r_{\lambda} / d r_{\lambda^{\prime}}$ & $\begin{array}{c}\text { Intercept on } \\
r_{\lambda^{\prime}}=\mathrm{Pb}^{206} / \mathrm{U}^{238} \\
\text { Axis of Asymptotic } \\
\text { Diffusion Curve }\end{array}$ \\
\hline 1.0 & 10.630 & 0.01191 \\
\hline 1.1 & 11.235 & 0.01432 \\
\hline 1.2 & 11.904 & 0.01729 \\
\hline 1.3 & 12.577 & 0.01985 \\
\hline 1.4 & 13.307 & 0.02273 \\
\hline 1.5 & 14.095 & 0.02592 \\
\hline 1.6 & 14.937 & 0.02929 \\
\hline 1.7 & 15.836 & 0.03282 \\
\hline 1.8 & 16.799 & 0.03654 \\
\hline 1.9 & 17.829 & 0.04043 \\
\hline 2.0 & 18.930 & 0.04445 \\
\hline 2.1 & 20.110 & 0.04866 \\
\hline 2.2 & 21.372 & 0.05270 \\
\hline 2.3 & 22.771 & 0.05825 \\
\hline 2.4 & 24.173 & 0.06215 \\
\hline 2.5 & 25.724 & 0.06692 \\
\hline 2.6 & 27.387 & 0.07184 \\
\hline 2.7 & 29.169 & 0.07688 \\
\hline 2.8 & 31.080 & 0.08207 \\
\hline 2.9 & 33.128 & 0.08734 \\
\hline 3.0 & 35.327 & 0.09280 \\
\hline 3.1 & 37.684 & \\
\hline 3.2 & 40.214 & \\
\hline 3.3 & 42.929 & \\
\hline 3.4 & 45.832 & \\
\hline 3.8 & 59.823 & \\
\hline 4.0 & 68.460 & \\
\hline 4.25 & 81.170 & \\
\hline 4.5 & 96.576 & \\
\hline
\end{tabular}

Asymptotic Behavior of Tilton's Continuous Diffusion Curves*

${ }^{*}$ Considering errors due to interpolation of various tables, the maximum percentage error is:

1. For $t \leq 2.5 \times 10^{9}$ years, values are correct to three decimal places.

2. For $t>2.5 \times 10^{9}$ years,

$$
\frac{\Delta\left(d r_{\lambda} / d r_{\lambda}{ }^{\prime}\right)}{d r_{\lambda} / d r_{\lambda}{ }^{\prime}} \leq 4.3 \times 10^{-5}
$$




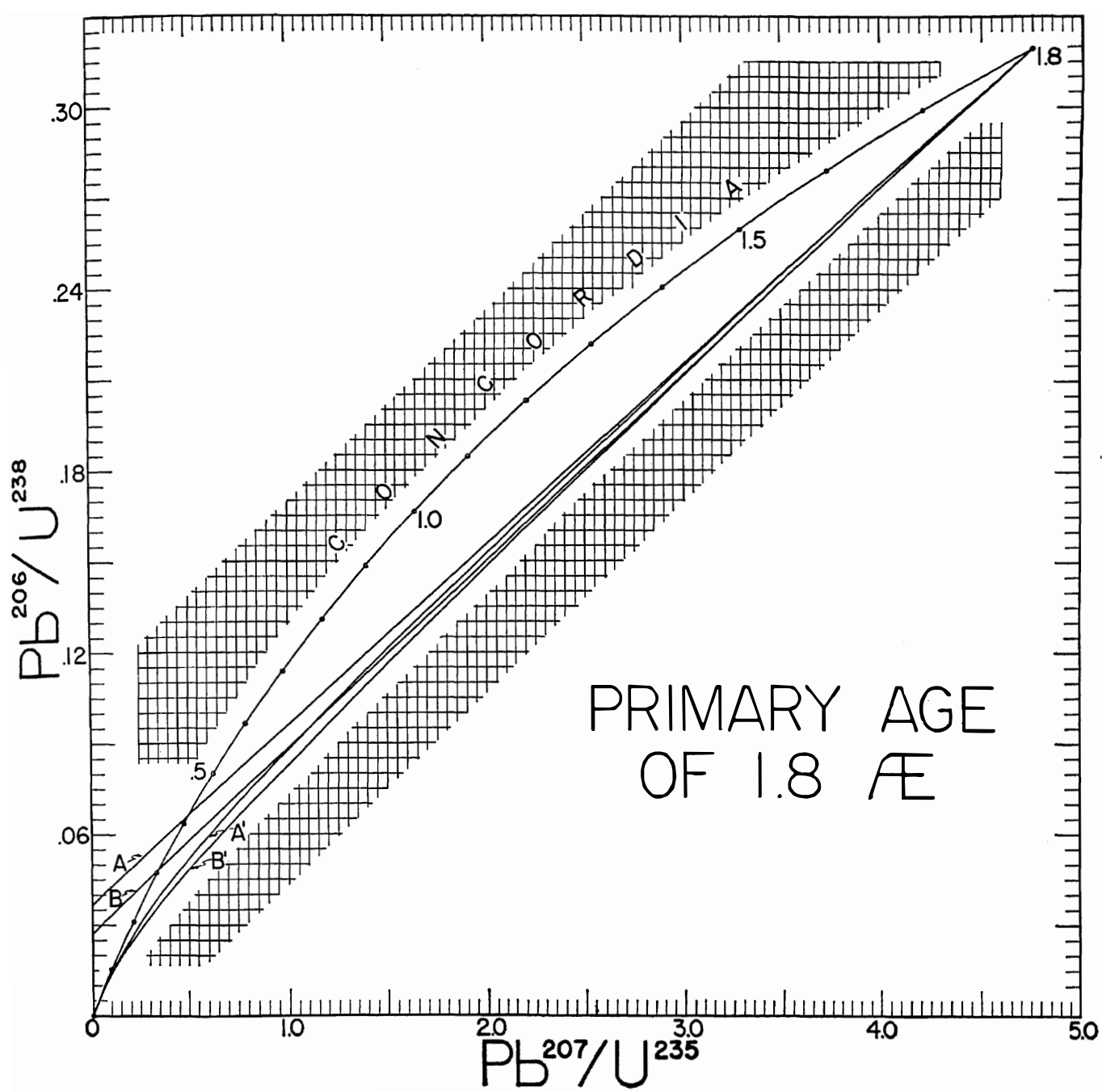

Fig. 3. Comparison of the continuous diffusion trajectories for $D(\tau)=D_{0}$ (a constant) [curve $A^{\prime}$ ] and $D(\tau)=D_{1} \tau\left(D_{1}\right.$ a constant) [curve $B^{\prime}$ ] for a primary age of 1800 m.y. Lines $A$ and $B$ are the extrapolated tangents to $A^{\prime}$ and $B^{\prime}$ drawn at the intersection of the diffusion trajectories with the concordia curve.

part of the curve. Figure 4 shows a curve for $\tau=2.5 \times 10^{9}$ years for $D=D_{1} \tau$ and a 'best' straight line passing through the linear region. The maximum per cent difference in $r_{\lambda}$ or $r_{\lambda}$. corresponding to the difference between the line and the curve is 1 per cent down to the value $r_{\lambda^{\prime}}=0.162$ (i.e. up to 73 per cent losses of $\mathrm{Pb}^{206}$ ).

The lower intercept on concordia corresponds to about 370 m.y. For the model $D=D_{0}$, the corresponding intercept is $530 \mathrm{~m} . \mathrm{y}$.

At $1.4 \times 10^{9}$ years the curve is linear to better than 1 per cent down to 73 per cent losses of $\mathrm{Pb}^{206}$ with an intercept of 180 to $200 \mathrm{~m} . \mathrm{y}$. Below the knee in the diffusion curve, the linear relationship fails.

If we plot the original data from uranium minerals used by Tilton, a satisfactory fit can be obtained for samples of all age groups with the $D_{1} \tau$ model. Because of the dispersion in these data due in part to the fact that these samples are not all the same age, it is at present impossible to distinguish these two diffusion mechanisms. The data obtained by Kuovo on some zircons from rapakivi granites from Finland are closest 


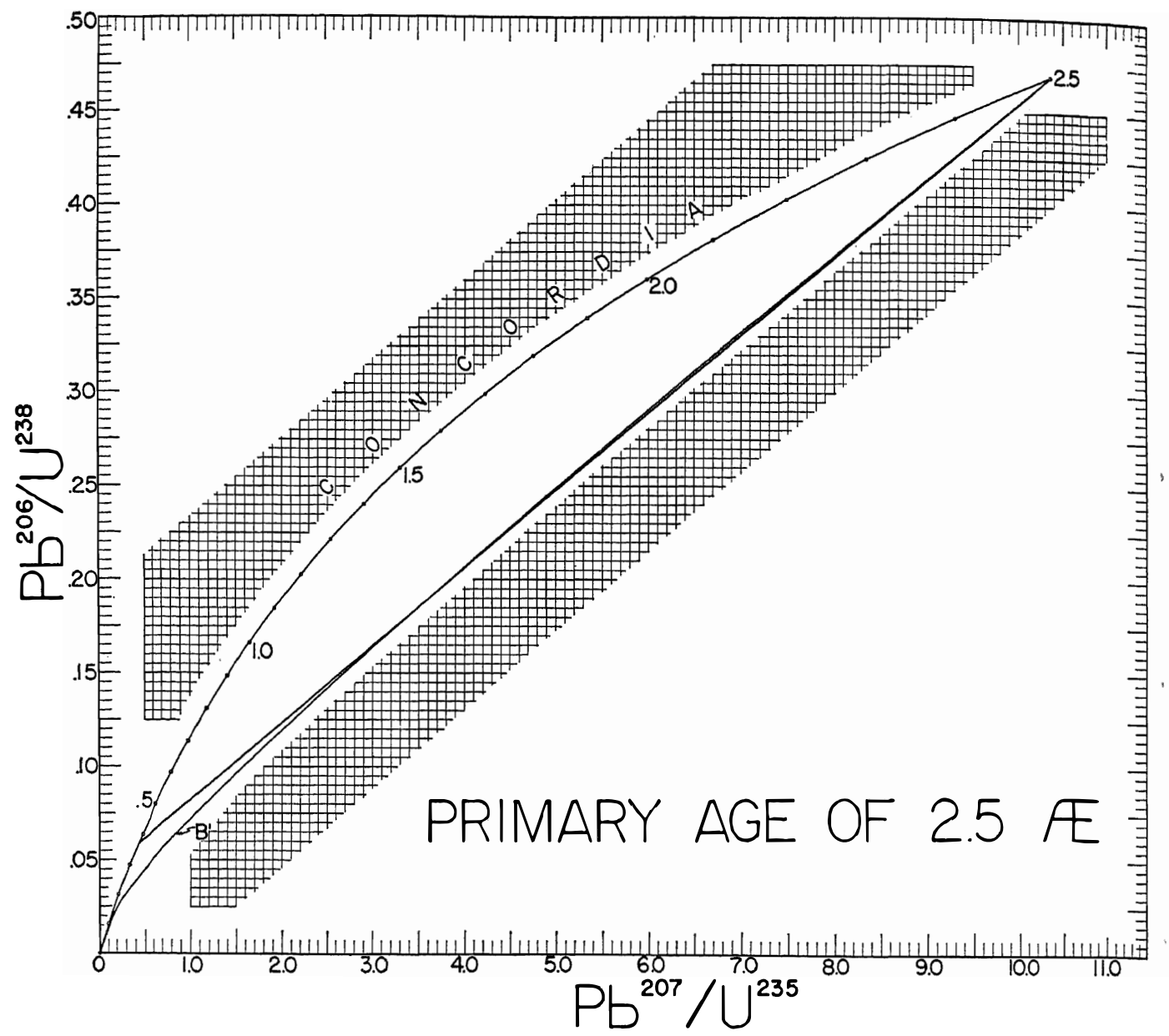

Fig. 4. Continuous diffusion trajectory for the radiation damage model $D(\tau)=D_{1} \tau$ and a 'best fit' straight line.

to a cotemporal suite. A plot of these data and the 1700-m.y. $D_{1} \tau$ curve are shown in Figure 5. A plot of the data for approximately cotemporal samples of about 1200 m.y. from Texas are shown in Figure 6 [after Wasserburg, Wetherill, Silver, and Flawn, 1962]. In the case of the Marble Mountain study by Silver and McKinney [1962] and Silver [1962] where the zircons were from the same rock we obtain good agreement between the line reported earlier by these workers and the best fit line for the irradiation damage ease. It thus appears that this model will satisfactorily explain the existing data discussed by Tilton [1960] and the newer, more detailed results by Silver [1962]. The case of the Johnny Lyon granodiorite does not fall in this category and appears to be episodic loss as discussed by Silver and Deutsch [1961].

The function $r_{\lambda}$ explicitly relates the daughterparent ratio to the diffusion history through the variable $T(\tau) / a^{2}$. In particular, for the radiation damage model, $T(\tau) / a^{2}$ is proportional to the uranium concentration. The function $f$ defined by $f \equiv r_{\lambda} /\left[e^{\lambda \tau}-1\right]$ is the daughter-parent ratio divided by the ratio for a closed system. In the case of no parent loss (or gain), $f$ is the fraction of daughter product retained. Silver [1962] plotted this function versus the uranium content for experimental results on suites of zircons from the Marble Mountains. He found that $f$ decreased with increasing uranium concentration. Theoretical curves of $f$ for different ages are shown in 


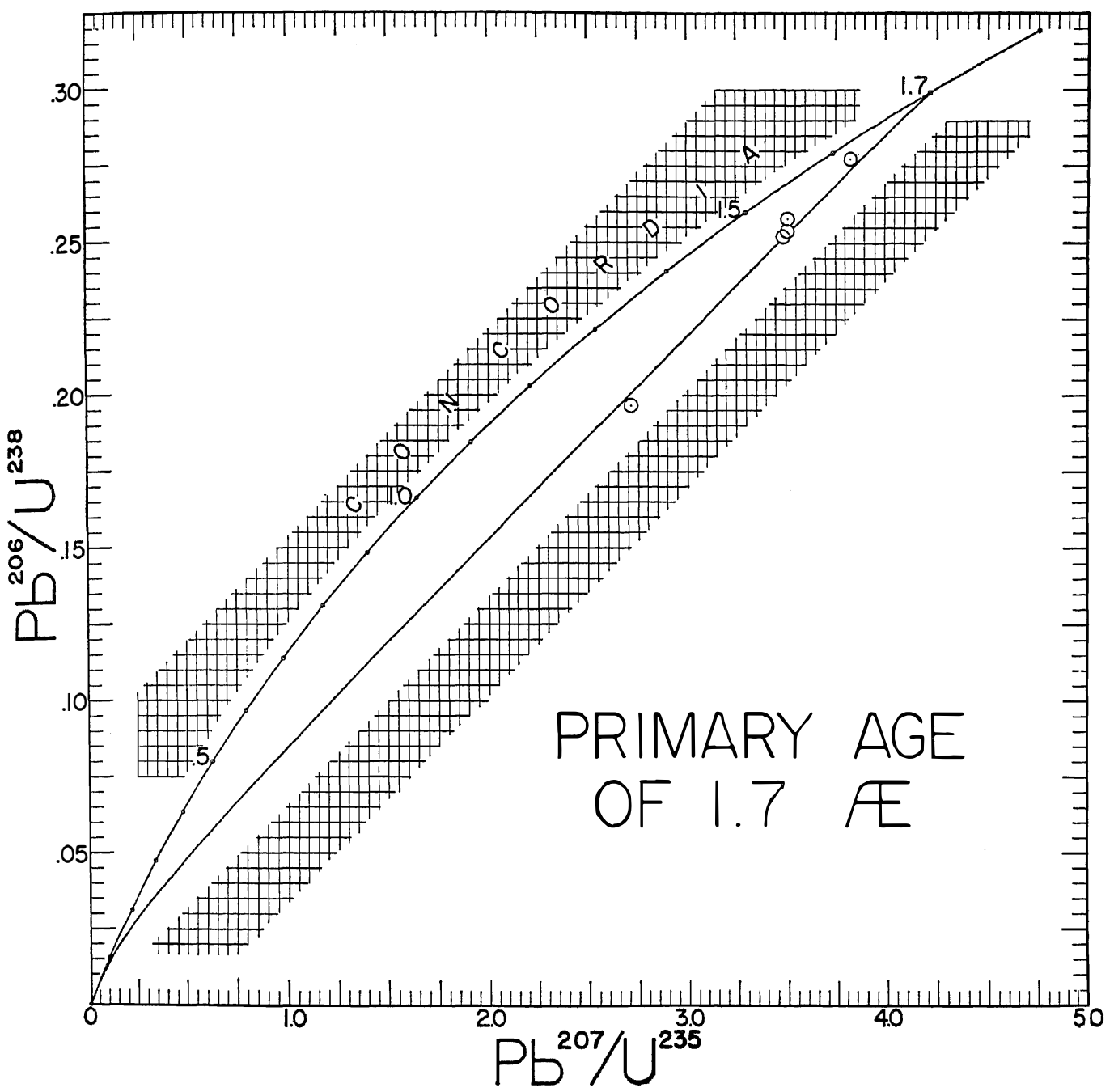

Fig. 5. Trajectory for $D(\tau)=D_{1} \tau$ for a primary age of 1700 m.y. The points represent the experimental results by Kuovo [1958] on zircons from some rapakivi granites from Finland. The open circles represent the errors.

Figure 7 for the model $D=D_{1} \tau$ and the saturation model $D=D_{0}$. The variables shown in the figure are $K$ and $\sqrt{D_{0}} / a$ for the two respective models. These two variables are proportional to the square root of the uranium concentration. From inspection of the curves it can be seen that, for $f>0.5, f \sim 1-$ constant $\sqrt{\bar{U}}$ for both cases. The exact dependence is given by the formula for $f$. To relate $f$ to the average uranium concentration it is necessary to determine $T(\tau) / U a^{2}$ for a sample. If we assume that a set of samples have identical characteristics, other than U content and radiation damage, then the value of $f$ for one of these samples determines $T(\tau) / a^{2}$ for the particular model. For the radiation damage models proposed, this determines $\sqrt{D_{0}} / a$ or $K$ for the sample. This then relates $f$ to $U$ for all the other samples, since the values of $\sqrt{D_{0}} / a$ or $K$ for the different samples are proportional to the $\sqrt{\mathrm{U}}$. From Figure 7 it is evident that the curves generated for the models $D_{0}$ and $D_{1} \tau$ are very similar and for the units chosen are almost identical at 2600 m.y. The other curves are very similar to within a scale factor. It will be difficult to distinguish these mechanisms on 


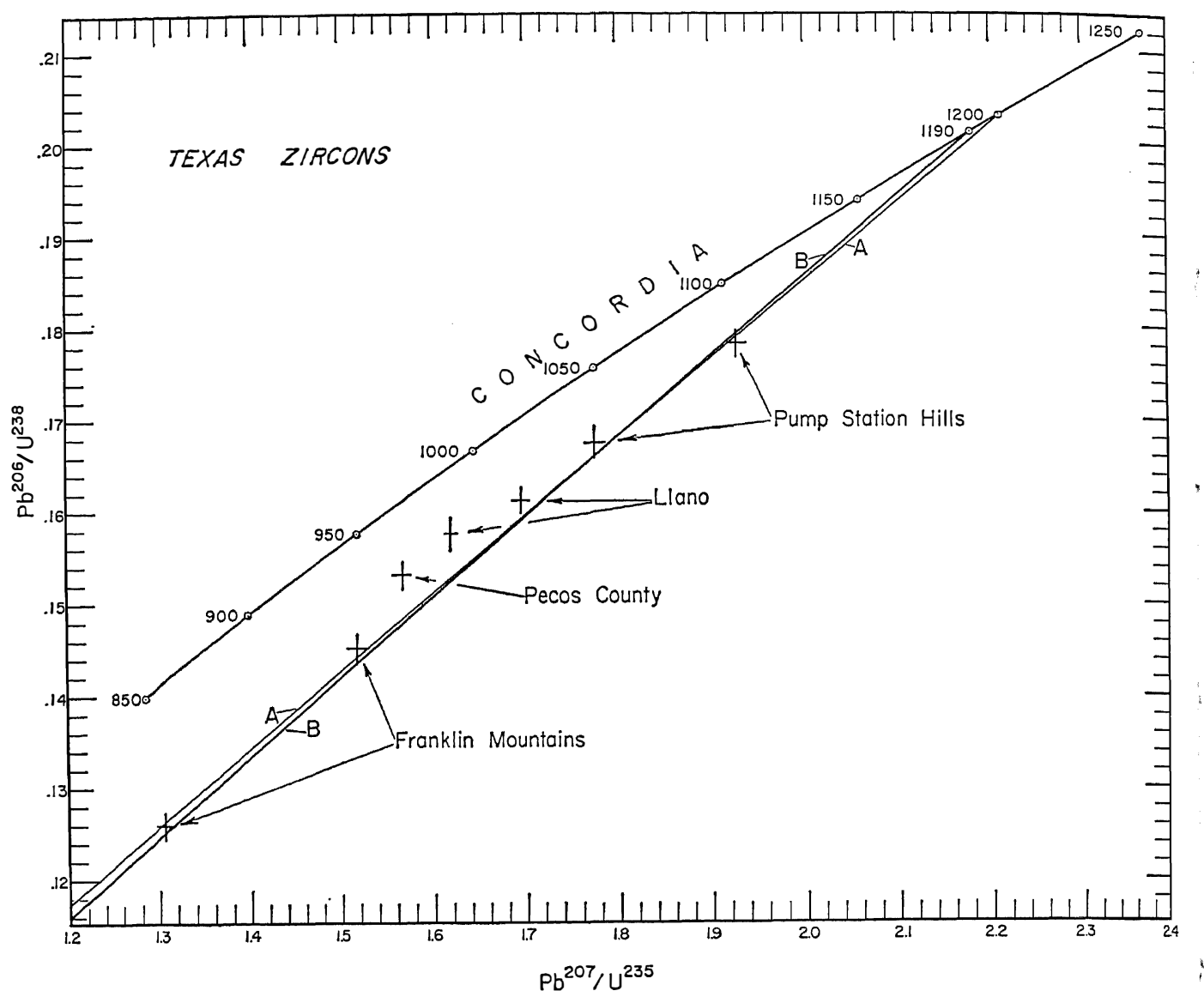

Fig. 6. Zircon data [after Wasserburg, Wetherill, Silver, and Flawn, 1962] for a suite of approximately contemporaneous granites from Texas. The fine line represents a 'best fit' chord. The heavy line is a continuous diffusion trajectory for $D(\tau)=D_{1} \tau$ for 1190 m.y.

the basis of the relationships between $f$ and $U$. The function $f$ for episodic diffusion loss is given in the appendixes in terms of the variable $T(\tau) / a^{2}$. The episodic case can also be considered in terms of a radiation damage model.

Silver et al. [1963], in a study of zircons from the Pacoima Canyon pegmatites, found essentially concordant $\mathrm{Pb}^{206} / \mathrm{U}^{238}$ and $\mathrm{Pb}^{207} / \mathrm{U}^{235}$ ages for zircons with extremely low uranium, thorium contents. The irradiation theory would appear to be in qualitative agreement with this aspect of the experimental data. However, the discordances of the rapakivi zircons analyzed by Kuovo [1958] do not show a regular relationship to uranium content, and the radiation damage as determined by X-ray spectrums does not follow a simple linear relationship. Holland and Gottfried [1955] have given a very detailed analysis of radiation damage to zircons and show that the unit cell dimensions are definitely not proportional to the total irradiation. These authors also have pointed out that some minerals, such as uraninites, that have suffered extreme irradiations do not manifest the degree of structural damage that would be present in zircons with a comparable history. This is in part manifest in the observation that uraninites very often give concordant lead-uranium ages. To reconcile the model with these facts it is necessary to assume that $D_{0}$ is dependent on the particular mineral structure and its impurities, and possibly that the characteristic diffusion distance $a$ is larger in uraninites than in zircons or monazites.

The functional form for $D$ that is proposed for the case of radiation damage can at present only be considered as a plausible model; the theory is 


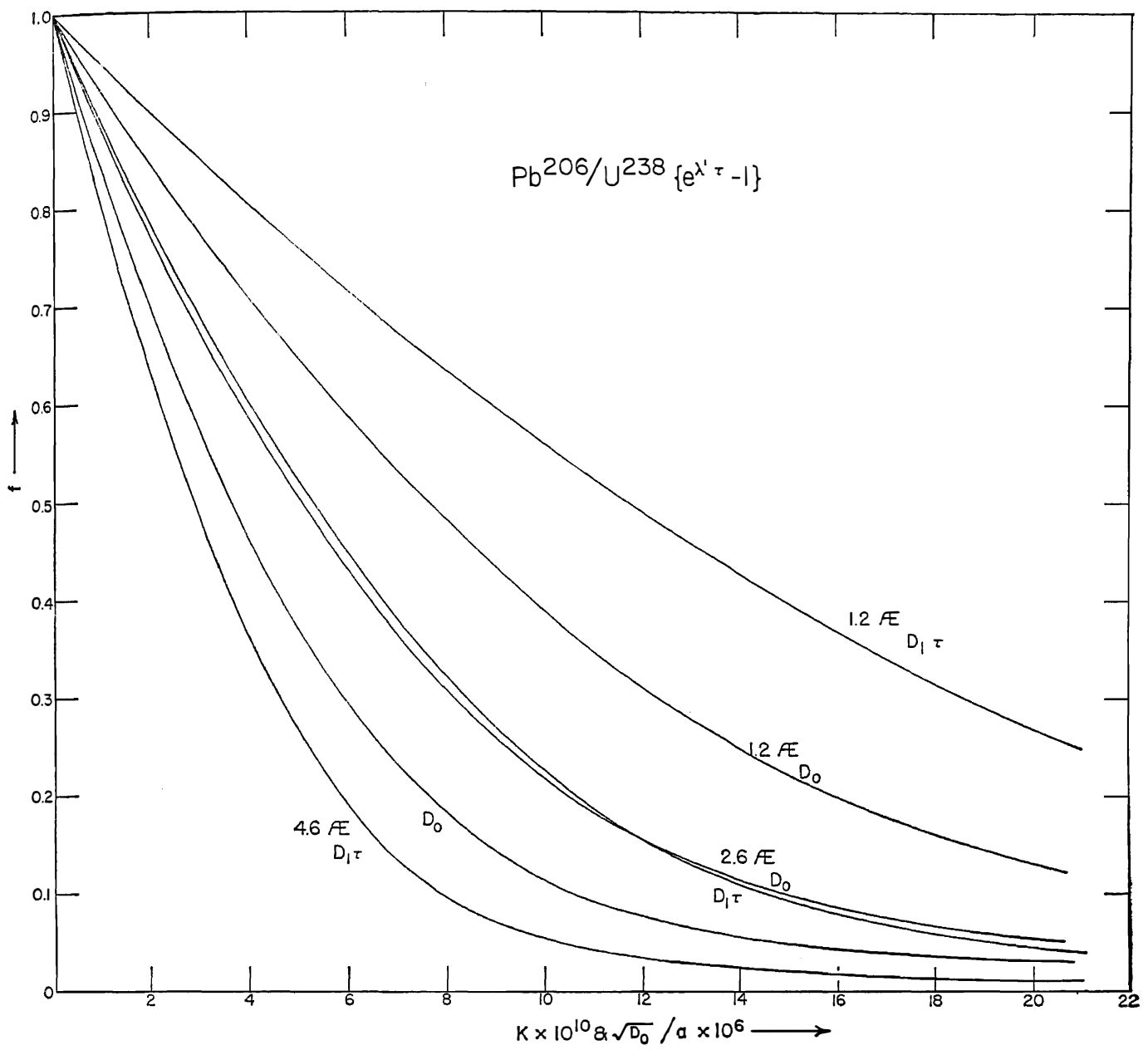

Fig. 7. $f=r_{\lambda^{\prime}} / e^{\lambda^{\prime} \tau}-1$ for $\lambda^{\prime}=1.54 \times 10^{-10} y^{-1}$ for the models $D(\tau)=D_{0}$ and $D(\tau)=D_{1} \tau$. The abscissa is $\sqrt{D_{0}} / a \times 10^{6}$ for the first model and $K \times 10^{10}=\pi \sqrt{2 D_{1}} / a \times 10^{10}$. These two parameters are proportional to the square root of the mean uranium concentration for the radiation damage model corresponding to saturation behavior and the proportional buildup, respectively.

thus rather phenomenological. Nonetheless this irradiation model appears to be in quantitative agreement with some of the existing experimental data.

If the term $D_{0}$ is not negligible, $D(\tau)=$ $D_{0}+D_{1} \tau$. It is to be expected that $D_{0} / D_{1}$ will vary within each grain and will therefore not be constant. In this case the daughter-parent ratios will be a function of both parameters and in general will not define a unique trajectory but instead a region bounded by the curves for $D(\tau)=D_{0}$ and $D(\tau)=D_{1} \tau$.

In the calculations presented here we have emphasized the part of the radiation damage curve before saturation. If the time $\tau$ is approximately $1 / b$, the diffusion coefficient will begin to saturate at the value $D_{1} / b$. In this intermediate time region the formula

$$
T(\tau)=\frac{D_{1}}{b}\left[\tau-\frac{1}{b}\left(1-e^{-b \tau}\right)\right]
$$

should be used. This means that the $r_{\lambda^{\prime}}, r_{\lambda}$ curves will depart from the curve corresponding to $D=D_{1} \tau$ and approach the curve for $D=D_{0}$ for saturation. These bounds are so strict that it does not appear necessary to evaluate the intermediate case. 
If the radiation damage is frozen in so that the defects have negligible mobility, it follows that the diffusion coefficient will not increase. If, however, the system is raised to a higher temperature at some later time, so that the mobility is significant, the diffusion coefficient will be increased. In this case the diffusion coefficient will (for periods short compared to the relaxation time) be proportional to the integrated radiation damage. As long as the intrinsic diffusion coefficient (i.e. without irradiation) is small, this can be treated as an episodic loss case where $D(\mu)=u \delta\left(\mu-\tau_{1}\right)$. Here $u$ is proportional to the integrated radiation damage (see appendix 2) and hence to the uranium concentration.

As discussed both by Tilton and in the present paper (see appendixes), the grain geometry does not play a significant role, and the conclusions for spherical geometry will not be altered by other choices (or mixtures) of simple grain shapes.

General continuous diffusion models. The general solution for daughter loss with any timedependent diffusion coefficient is given by (3). This includes the episodic case first discussed by Wetherill [1956]. If we restrict the functional form of $D(\tau)$ to a smooth monotonically increasing function of time, it is possible to draw rather general conclusions about the curve $r_{\lambda}[\tau, D(\tau)], r_{\lambda^{\prime}}[\tau, D(\tau)]$. From the preceding discussion it is quite evident that the linear part of the curves is rather closely approximated by the asymptotic lines when

$$
\int_{0}^{r} D(\eta) d \eta \ll a^{2}
$$

As is shown in the appendix, the slope of this line $d r_{\lambda} / d r_{\lambda^{\prime}}$ at the intersection with concordia is only dependent on the diffusion law.

Figure 8 shows $d r_{\lambda} / d r_{\lambda^{\prime}}$ for different diffusion laws $D(\tau)=D_{0}, D(\tau)=D_{1} \tau^{1}, D(\tau)=D_{2} \tau^{2}$, $D(\tau)=D_{3} \tau^{3}$. The slopes on a $\mathrm{Pb}^{206} / \mathrm{U}^{238}$, $\mathrm{Pb}^{207 / \mathrm{U}^{235}}$ diagram continuously increase and tend toward the limiting case of modern $(\tau=0)$ episodic loss. The general tendency is thus to restrict the particular diffusion curve to a narrower region of the $r_{\lambda}, r_{\lambda}$, diagram for higher powers of the exponent. This tends to make the exact behavior of the curve more linear. In general, for polynomials with positive coefficients the curve will lie between the modern episodic loss line and the asymptote for the corresponding lowest power of $\tau$ in the polynomial. For any continuous diffusion model where $D(\tau)$ is a smooth monotonically increasing function, the curve will be similar to the case discussed by Tilton. The degree of curvature in the 'linear' region will depend on the relative magnitudes of the terms. All the curves will show a relatively linear behavior in the neighborhood of concordia and will have a rapid change of slope and curvature in the neighborhood of the origin. These curves must all be asymptotic to the concordia curve at the origin.

The application of this theory to experiments is complicated by the fact that any mineral sample will have a variety of grains and mosaic structures that may act as free surfaces where $C(r, \tau)$ effectively equals zero. It follows that any sample will contain a mixture of 'grains' with different uranium concentrations and characteristic diffusion lengths $(a)$. The accessible region on the $r_{\lambda}, r_{\lambda^{\prime}}$ diagram will thus lie in the area indicated in Figure 9. If this condition obtained, it would be impossible to deduce an age from a single sample, even if $D(\tau)$ were known. The fact that the experimental results

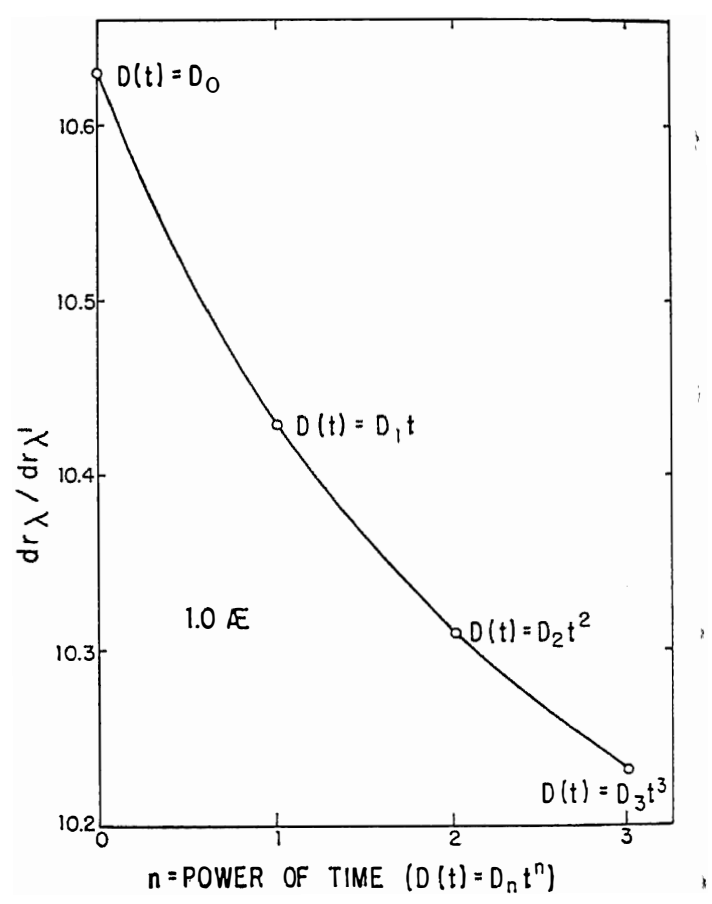

Fig. 8. The asymptotic slope $d r_{\lambda} / d r_{\lambda^{\prime}}$ at 1000 m.y. for the cases $D(\tau)=D_{n} \tau^{n}$ where $D_{n}$ is a constant. $\lambda=9.72 \times 10^{-10} \mathrm{y}^{-1}, \lambda^{\prime}=1.54 \times 10^{-10} \mathrm{y}^{-1}$. 


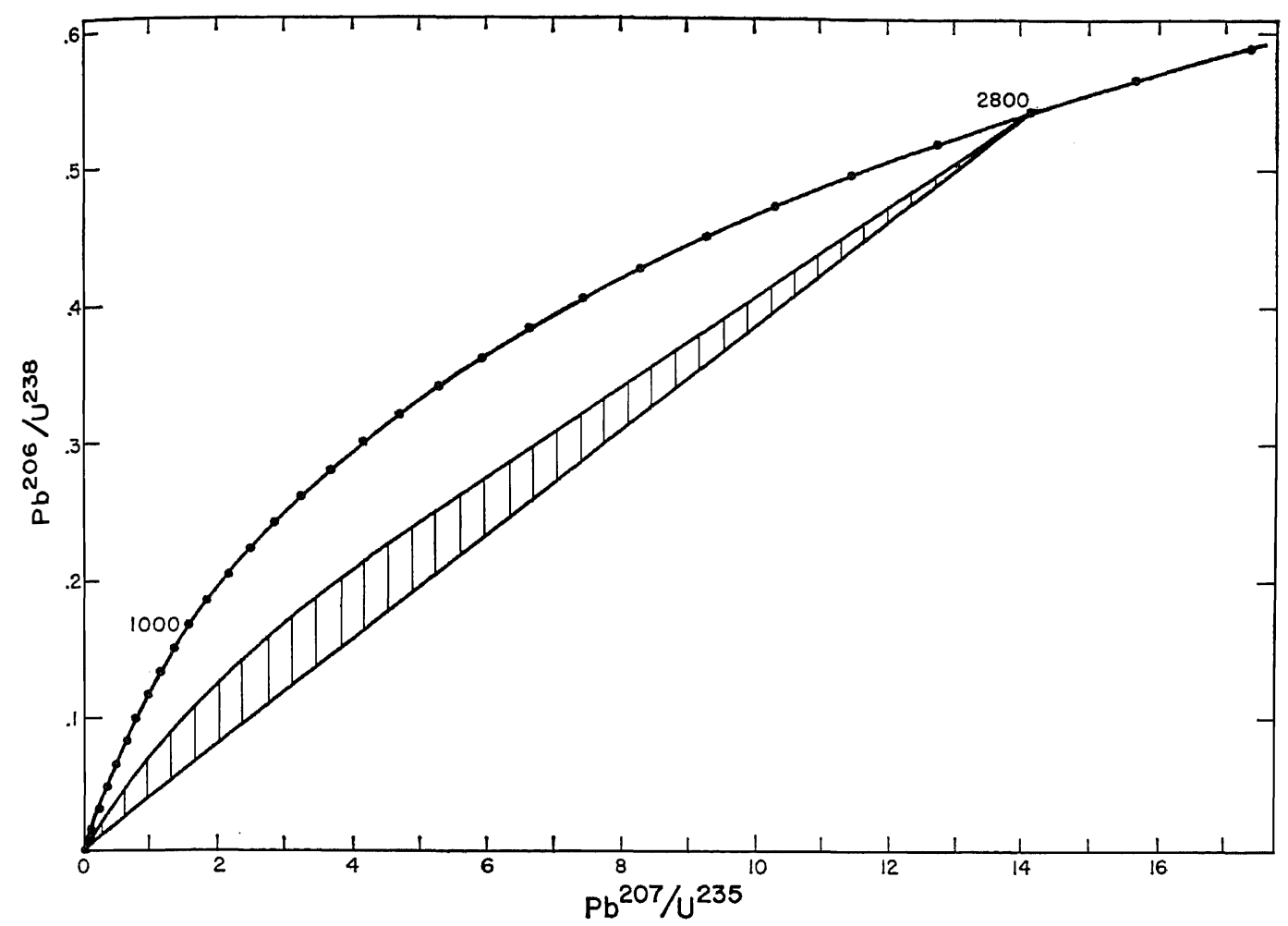

Fig. 9. The accessible region on the $r_{\lambda}, r_{\lambda^{\prime}}$ plane for a given continuous diffusion law. All possible mixtures of samples with arbitrary values of the diffusion parameter must lie in the hatched region. If $D(\boldsymbol{\tau})$ were zero from the present until $\tau_{1}$ years ago, the hatched region would be displaced and would connect with the concordia curve at the point corresponding to $\tau_{1}$ instead of at the origin.

lie on the linear parts of a continuous diffusion trajectory indicates that the distribution of lead and uranium in the minerals is in domains with relatively small values of $\int_{0} \tau D(\eta) d \eta / a^{2}$ (less than $\sim 0.2$ ). Any mixture of such grains will therefore lie on the linear part of the diffusion trajectory and determine a unique age. It is also well known that zircons may show a distinct zonation in uranium concentration, whereas in the formal problem treated here the concentration was assumed to be uniform. We must conclude from the experimental observations that the 'grain size' which determines the diffusion length is much less than the distances over which the uranium concentration changes significantly.

We have discussed cases for the boundary condition $C(r, \tau)=0$ on the surface. In using this boundary condition we have assumed that any daughter product which reaches this surface is removed from the system. There is at present no strong evidence that surficial radiogenic lead is on the surfaces of zircon crystals, insofar as it is leachable by acid treatment. Nonetheless, it is important to observe that for small values of the diffusion parameter, if all the diffused daughter products were accumulated on the grain boundaries until recent times and then were lost in varying amounts, the values of $r_{\lambda}, r_{\lambda}$. (including the remaining grain boundary material) would still lie on the linear part of the diagram. This follows because the total material lost must lie on the straight line extended through the concordia curve.

The problem we have so far treated ignores the possibility of parent diffusion. This question was first discussed by Wetherill [1963]. The solution to this problem (equations 1 and 2) can be obtained for a variety of geometries (see appendixes). The result for the spherical case is 


$$
r_{\lambda}=\lambda e^{\lambda \tau} \frac{\sum_{n=1}^{\infty} \frac{1}{n^{2}} \int_{0}^{\tau} \exp \left\{-n^{2} \pi^{2}\left[T(\tau)-T(\mu)+T^{*}(\mu)\right] / a^{2}-\lambda \mu\right\} d \mu}{\sum_{m=1}^{\infty} \frac{1}{m^{2}} \exp \left[-m^{2} \pi^{2} T^{*}(\tau) / a^{2}\right]}
$$

Here

and

$$
T(\tau)=\int_{0}^{\tau} D(\eta) d \eta
$$

$$
T^{*}(\tau)=\int_{0}^{\tau} D^{*}(\eta) d \eta
$$

are the integrals of the diffusion coefficients for daughter and parent, respectively. This result therefore represents a rather general case, including episodic loss of daughter and parent. For the latter case

$$
\begin{aligned}
& D(\tau)=\sum_{i} u_{i} \delta\left(\tau-\tau_{i}\right) \\
& D^{*}(\tau)=\sum_{i} u_{i}{ }^{*} \delta\left(\tau-\tau_{i}\right)
\end{aligned}
$$

The qualitative results for the continuous diffusion of both parent and daughter are similar to that obtained for pure daughter diffusion. For the case $D(\tau)=D^{*}(\tau)$, i.e. identical diffusion coefficients of daughter and parent, $r_{\lambda}=e^{\lambda \tau}-1$, and the resulting ages are concordant independent of the diffusion history. When $D^{*}(\tau)>D(\tau)$ the diffusion trajectory is above the concordia curve.

The diffusion trajectory for pure daughter loss lies beneath the concordia curve and is linear in the vicinity of this curve. The diffusion trajectory for pure parent loss lies above the concordia curve and is also linear in the vicinity of concordia. If $D^{*}(\tau)$ is proportional to $D(\tau)$, then in the neighborhood of concordia the diffusion trajectory for simultaneous diffusion will lie on a straight line. The slope of this line will be between the slope for pure daughter loss for the function $D(\tau)$ and the slope for pure parent loss for $D^{*}(\tau)$. On a normal $\mathrm{Pb}^{208} / \mathrm{U}^{238}, \mathrm{~Pb}^{207} / \mathrm{U}^{235}$ diagram the slope

$$
\frac{d \mathrm{~Pb}^{206} / \mathrm{U}^{238}}{d \mathrm{~Pb}^{207} / \mathrm{U}^{235}}
$$

will be greater for pure daughter loss than for pure parent loss (see Figure 10), the intermediate cases having continuously decreasing slope. Figure 11 shows the slope in the neighborhood of concordia as a function of the ratio $k=D^{*}(\tau) / D(\tau)$ for two diffusion laws. It is evident that a relatively small proportion of parent diffusion causes a significant change in the slope, a large change occurring for $k$ between 0 and 0.1 . Since the slope decreases with increasing parent diffusion, the effect is to pull the continuous diffusion curve further from the modern episodic loss line. Consequently the trajectory for continuous diffusion of parent and daughter is more curved than the corresponding case of pure daughter loss, since the diffusion trajectory must pass through the origin (for $k<1$ ).

In any diffusion history where $D^{*}(\tau)$ is pro- : portional to $D(\tau)$ the trajectory is a straight line in the neighborhood of concordia, and this line intersects concordia at the primary age. It therefore follows that the primary age is correctly defined by the standard construction procedures in use for a wide variety of diffusion histories.

It is seen that a wide variety of continuous diffusion histories will generate trajectories on the $r_{\lambda^{\prime}}, r_{\lambda}$ diagram which are somewhat like the model proposed by Tilton [1960]. Rather considerable fluctuations in the behavior of $D(\tau)$ will not alter the gross behavior, the case of strongly peaked events being the main exception.

The mathematical details of the theory presented in this paper are given in the appendixes. The asymptotic theory developed gives

$$
\frac{d r_{\lambda}}{d r_{\lambda^{\prime}}}=\frac{\lambda e^{\lambda \tau} \int_{0}^{\tau} d \mu e^{-\lambda \mu}\left\{\sqrt{T^{*}(\mu)-T(\mu)+T(\tau)}-\sqrt{T^{*}(\tau)}\right\}}{\lambda^{\prime} e^{\lambda^{\prime} \tau} \int_{0}^{\tau} d \mu e^{-\lambda^{\prime} \mu}\left\{\sqrt{T^{*}(\mu)-T(\mu)+T(\tau)}-\sqrt{T^{*}(\tau)}\right\}}
$$




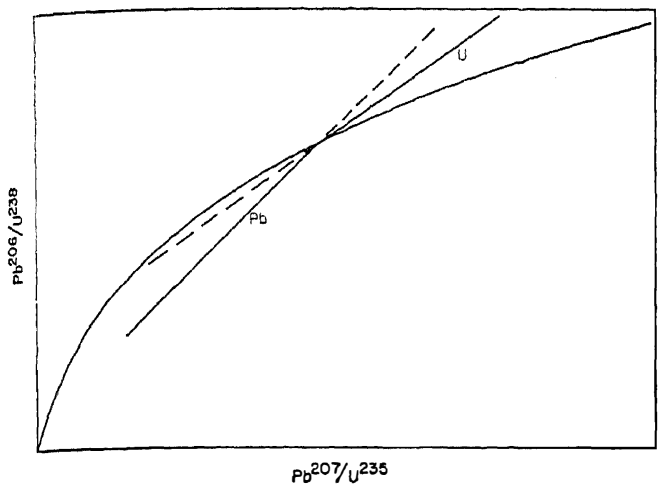

Fig. 10. The asymptotic behavior of pure lead and pure uranium diffusion with the same diffusion law. The slope for pure lead diffusion is greater than that for pure uranium diffusion. Mixed lead and uranium diffusion will have intermediate slopes.

This theory permits the calculation of a large class of trajectories in the neighborhood of concordia by very elementary means, and makes it possible to estimate the nature of the curves without excessive computation.
Conclusions. The ratio $r_{\lambda}$ of radiogenic daughter to residual parent was investigated for a continuous diffusion model for a diffusion coefficient which is an arbitrary function of time. A solution for simultaneous diffusion of both parent and daughter is given in closed form. An asymptotic theory is also developed which describes the trajectories $r_{\lambda}, r_{\lambda}$, for paired decay schemes (with decay constants $\lambda$ and $\lambda^{\prime}$ ) in the neighborhood of concordia. A wide class of systems is shown to be linear in the $r_{\lambda}, r_{\lambda^{\prime}}$, diagram when $\int_{0} \tau D(\eta) d \eta / a^{2} \ll 1$.

A radiation damage model is proposed for the diffusion coefficient, which relates $D(\tau)$ to the integrated irradiation damage, and to the uranium (and thorium) contents. The functional relationship between $r_{\lambda}$ and the uranium content is found for three important cases. The model $D(\tau)=D_{1} \tau$ yields a $r_{\lambda}, r_{\lambda}$, curve very similar to that obtained by Tilton for $D=D_{0}$. The slope is somewhat greater and the linear region more extended. The theoretical curves are found to be in reasonable agreement with the existing experimental data.

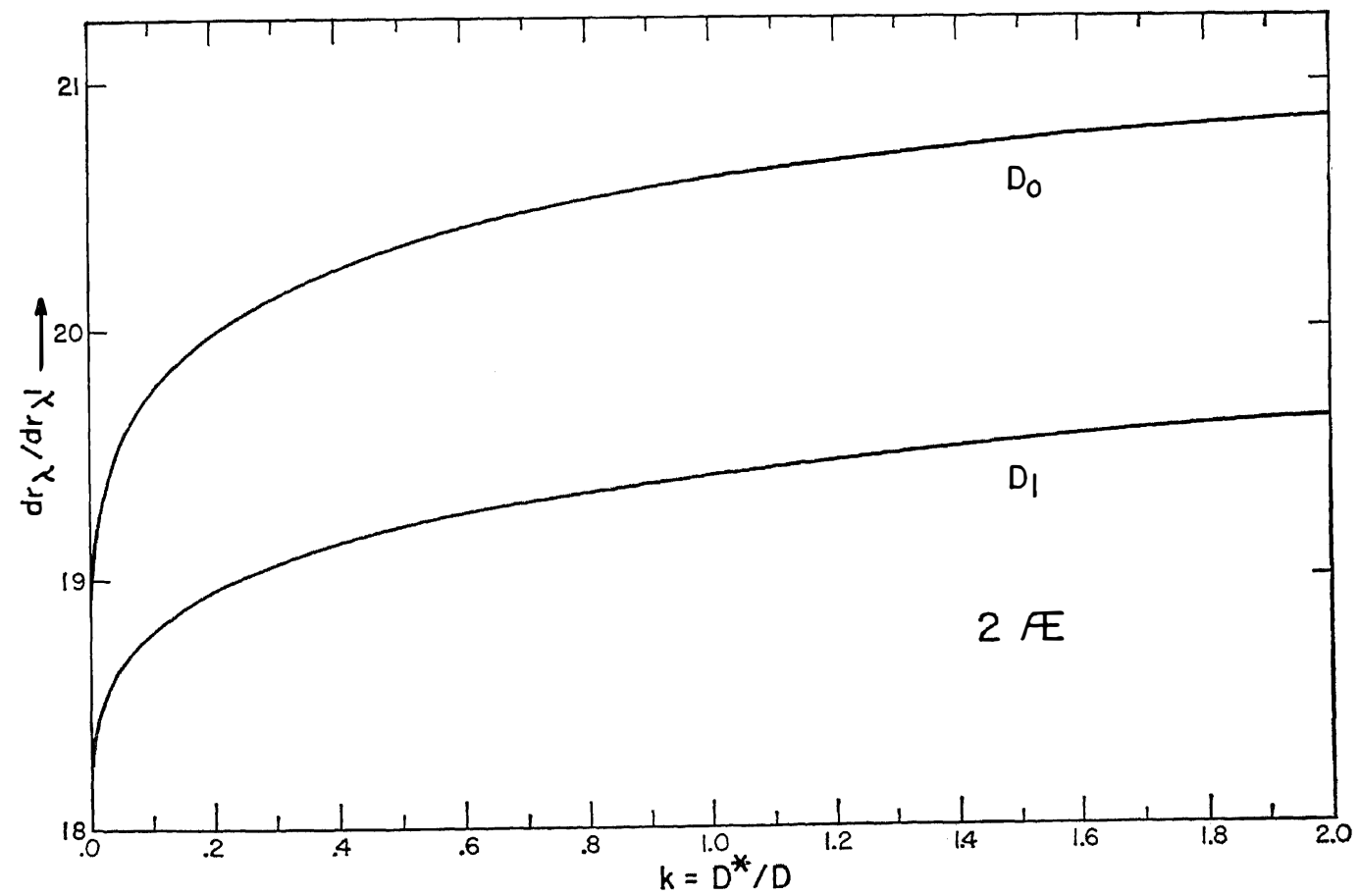

Fig. 11. The slope $d r_{\lambda} / d r_{\lambda^{\prime}}$ for the case $D(\tau)=k D^{*}(\tau)$ for the models $D(\tau)=D_{0}$ and $D_{1} \tau$, respectively. The slope changes rapidly for small amounts of uranium diffusion. $\lambda=9.72 \times 10^{-10} \mathrm{y}^{-1}$, $\lambda^{\prime}=1.54 \times 10^{-10} \mathrm{y}^{-1}$. 


\section{APPENDIX 1}

Theorem 1. Consider the operator

$$
L \equiv(\partial / \partial \tau)-D(\tau) \nabla^{2}
$$

Following Crank [1956], define

$$
T(\tau)=\int_{0}^{\tau} D(\eta) d \eta
$$

for $D(\eta) \geq 0$; then

$$
L=D(\tau)\left[(\partial / \partial T)-\nabla^{2}\right]
$$

Let $f(\mathrm{r}, T(\tau))$ be a solution of $L f=0$ such that $f$ vanishes on a surface $\sigma$ for all time, and $f(\mathbf{r}, 0)=1$. Define

$$
F(\mathrm{r}, \tau, \mu)= \begin{cases}0 & \tau<\mu \\ \phi(\mu) f(\mathrm{r}, T(\tau)-T(\mu)) & \tau \geq \mu\end{cases}
$$

Then $L F=0$ and $F(\mathbf{r}, \mu, \mu)=\phi(\mu)$. The solution to $L C(\mathbf{r}, \tau)=\phi(\tau)$, with the initial condition $C(\mathrm{r}, 0)=0$ and boundary condition $C(\mathrm{r}, \tau)=0$ on $\sigma$ is then given by

$$
\begin{aligned}
C(\mathbf{r}, \tau)=\int_{0}^{\tau} \phi(\mu) f(\mathrm{r}, T(\tau) & -T(\mu)) d \mu \\
& =\int_{0}^{\tau} F(\mathbf{r}, \tau, \mu) d \mu
\end{aligned}
$$

Functions $f(r, \tau)$ for a variety of geometries can be found in Carslaw and Jaeger [1959].

For the case of diffusion from a sphere with only daughter diffusion ${ }^{1}$

$$
\begin{gathered}
\partial C_{\lambda}(r, \tau) / \partial \tau=D(\tau) \nabla^{2} C_{\lambda}(r, \tau)+\lambda U_{0} e^{-\lambda \tau} \\
C(a, \tau)=0 \quad C(0, \tau)=0
\end{gathered}
$$

Following theorem 1 we have for $\mu \leq \tau$

$$
\begin{gathered}
F(r, \tau, \mu)=\frac{2 a \lambda \mathrm{U}_{0}}{\pi r} \sum_{n=1}^{\infty} \frac{(-1)^{n+1}}{n} \sin (n \pi r / a) \\
\cdot \exp \left\{-\pi^{2} n^{2}[T(\tau)-T(\mu)] / a^{2}-\lambda \mu\right\} \\
C_{\lambda}(r, \tau)=\frac{2 a \lambda \mathrm{U}_{0}}{\pi r} \sum_{n=1}^{\infty} \frac{(-1)^{n+1} \sin (\mathrm{n} \pi r / a)}{n} \\
\cdot \int_{0}^{\tau} \exp \left\{-\pi^{2} n^{2}[T(\tau)-T(\mu)] / a^{2}-\lambda \mu\right\} d \mu
\end{gathered}
$$

1 A preliminary version of the formal theory given in this paper for pure daughter loss has been presented [Wasserburg, 1962].

$$
\begin{aligned}
& r_{\lambda}=\frac{6 \lambda}{\pi^{2}} \exp \lambda \tau \sum_{n=1}^{\infty} \frac{1}{n^{2}} \\
& \cdot \int_{0}^{r} \exp \left\{-n^{2} \pi^{2}[T(\tau)-T(\mu)] / a^{2}-\lambda \mu\right\} d \mu
\end{aligned}
$$

The case $D(\tau)=\Sigma_{j} u_{j} \delta\left(\tau-\tau_{j}\right)$, where the $u_{i}$ are constants, corresponds to the model of episodic lead loss as discussed by Wetherill [1956]. In the present case, however, no loss or gain of uranium is taken into consideration.

For the case $D(\tau)=D_{1} \tau, r_{\lambda}$ can be written in the form

$$
\begin{aligned}
r_{\lambda}(\tau) & =\frac{6 \lambda \tau}{\pi^{2}} \sum_{n=1}^{\infty} \frac{1}{n^{2}} \\
& \cdot \int_{0}^{1} \exp \left\{y\left[\lambda \tau-\frac{K^{2} n^{2} \tau^{2}}{4}(2-y)\right]\right\} d y
\end{aligned}
$$

where $K=\pi \sqrt{2 D_{1}} / a$.

For the case of an infinite circular cylinder of radius $a$

$$
\begin{aligned}
& C_{\lambda}= \frac{2 \lambda U_{0}}{a} \sum_{n=1}^{\infty} \frac{J_{0}\left(r \alpha_{n}\right)}{\alpha_{n} J_{1}\left(a \alpha_{n}\right)} \\
& \cdot \int_{0}^{\tau} \exp \left\{-\lambda \mu-\alpha_{n}{ }^{2}[T(\tau)-T(\mu)]\right\} d \mu \\
& r_{\lambda}= 4 \lambda \sum_{n=1}^{\infty} \frac{1}{\left(\alpha_{n} a\right)^{2}} \\
& \cdot \int_{0}^{\tau} \exp \left\{\lambda(\tau-\mu)-\alpha_{n}{ }^{2}[T(\tau)-T(\mu)]\right\} d \mu
\end{aligned}
$$

where $\alpha_{n} a$ are the roots of $J_{0}\left(\alpha_{n} a\right)=0$.

Asymptotic theory for daughter loss. For the asymptotic behavior, consider the diffusion of a radiogenic daughter from a half-space. The radioactive parent is taken to be uniformly distributed.

$$
\begin{gathered}
\frac{C_{\lambda}(0, \tau)=0}{\downarrow x} x=0 \\
\frac{\partial C_{\lambda}(x, \tau)}{\partial \tau}=D(\tau) \nabla^{2} C_{\lambda}(x, \tau)+\lambda U_{\lambda}(\tau)
\end{gathered}
$$

Let the initial condition be $C_{\lambda}(x, 0)=0$ and the boundary condition be $C_{\lambda}(0, \tau)=0$. Following the theorem we have

$$
f(x, \tau)=\operatorname{erf}\left[\frac{x}{2 \sqrt{T(\tau)}}\right]
$$

and 
$F(x, \tau, \mu)=\lambda U_{\lambda}(\mu) \operatorname{erf}\left[\frac{x}{2 \sqrt{T(\tau)-T(\mu)}}\right]$

Hence

$C_{\lambda}(x, \tau)=\lambda \int_{0}^{\tau} U_{\lambda}(\mu) \operatorname{erf} \frac{x}{2 \sqrt{T(\tau)-T(\mu)}} d \mu$

or if $U_{\lambda}(\tau)=U_{\lambda^{0}} \exp -\lambda \tau$

$C_{\lambda}(x, \tau)=\lambda U_{\lambda}{ }^{0}$

$\cdot \int_{0}^{\tau} \exp \{-\lambda \mu\} \operatorname{erf}\left[\frac{x}{2 \sqrt{T(\tau)-T(\mu)}}\right] d \mu$

The flux

$J_{\lambda}(x, \tau)=-D(\tau) \frac{\partial C_{\lambda}(x, \tau)}{\partial x}=\frac{-\lambda U_{\lambda}^{0} D(\tau)}{\sqrt{\pi}}$

$\cdot \int_{0}^{\tau} \frac{\exp \{-\lambda \mu\} \exp \left[-x^{2} / 4(T(\tau)-T(\mu))\right] d \mu}{\sqrt{T(\tau)-T(\mu)}}$

and at $x=0$

$$
\begin{aligned}
J_{\lambda}(0, \tau)= & \frac{-\lambda U_{\lambda}{ }^{0} D(\tau)}{\sqrt{\pi}} \int_{0}^{\tau} \frac{\exp \{-\lambda \mu\} d \mu}{\sqrt{T(\tau)-T(\mu)}} \\
= & \frac{-\lambda U_{\lambda}{ }^{0} 2}{\sqrt{\pi}} \frac{d}{d \tau} \\
& \cdot \int_{0}^{\tau} \exp \{-\lambda \mu\} \sqrt{T(\tau)-T(\mu)} d \mu
\end{aligned}
$$

If $Q_{\lambda}$ be the net loss of material per unit of surface area at time $\tau$

$$
\begin{aligned}
Q_{\lambda} & =\int_{0}^{\tau} J_{\lambda}(0, \mu) d \mu \\
& =\frac{\lambda U_{\lambda}{ }^{0} 2}{\sqrt{\pi}} \int_{0}^{\tau} \exp \{-\lambda \mu\} \sqrt{T(\tau)-T(\mu)} d \mu
\end{aligned}
$$

If we consider the case of diffusion from a grain for which

$$
\int_{0}^{\tau} D(\eta) d \eta \ll a^{2}
$$

where $a$ is a characteristic radius of curvature for the grain, we can calculate the net loss of daughter product using (1). Let the total surface area of the grain be $\sigma$ and the volume be $V$. Then the ratio of daughter to parent at time $\tau$ is

$$
\begin{array}{r}
r_{\lambda} \equiv \frac{V U_{\lambda}(\tau) e^{\lambda \tau}-V U_{\lambda}(\tau)-\sigma Q_{\lambda}}{V U_{\lambda}(\tau)} \\
=e^{\lambda \tau}-1-\frac{\sigma e^{\lambda \tau}}{V} \frac{\lambda 2}{\sqrt{\pi}} \\
\cdot \int_{0}^{\tau} e^{-\lambda \eta} \sqrt{T(\tau)-T(\eta)} d \eta
\end{array}
$$

Let us suppose the diffusion coefficient $D(\tau)$ can be written in the form $D(\tau)=\gamma \mathscr{D}(\tau)$, where $\gamma$ is an arbitrary dimensionless parameter. Then we can write

$$
T(\tau)=\gamma J(\tau)=\gamma \int_{0}^{\tau} D(\eta) d \eta
$$

The various values for $C_{\lambda}(\mathbf{r}, \tau)$ and $r_{\lambda}(\tau)$ corresponding to different values for $\gamma$ will represent the concentration and daughter-parent ratio for similar diffusion histories differing only in the scale factor for $D(\tau)$. We can then write for a fixed time $\tau$ :

$\frac{d r_{\lambda}}{d \gamma}=\frac{\sigma \lambda e^{\lambda \tau}}{V \sqrt{\pi} \gamma^{1 / 2}} \int_{0}^{\tau} e^{-\lambda \mu} \sqrt{J(\tau)-J(\mu)} d \mu$

For another parent $U_{\lambda^{\prime}}$ with decay constant $\lambda^{\prime}$, a similar equation will hold. If the daughter product has the identical diffusion law

$$
\begin{aligned}
& \frac{d r_{\lambda^{\prime}}=\frac{\sigma \lambda^{\prime} e^{\lambda^{\prime} \tau}}{d \gamma}}{V \sqrt{\pi} \gamma^{1 / 2}} \\
& \quad \cdot \int_{0}^{\tau} e^{-\lambda^{\prime} \gamma} \sqrt{\Im(\tau)-J(\gamma)} d \gamma
\end{aligned}
$$

it therefore follows that

$$
\begin{array}{r}
\frac{d r_{\lambda}}{d r_{\lambda^{\prime}}}=\frac{\lambda e^{\lambda \tau} \int_{0}^{\tau} e^{-\lambda \mu} \sqrt{J(\tau)-J(\mu)} d \mu}{\lambda^{\prime} e^{\lambda^{\prime} \tau} \int_{0}^{\tau} e^{-\lambda^{\prime} \mu} \sqrt{J(\tau)-J(\mu)} d \mu} \\
=\frac{e^{\lambda \tau} \lambda \int_{0}^{\tau} e^{-\lambda \mu} \sqrt{T(\tau)-T(\mu)} d \mu}{e^{\lambda^{\prime} \tau} \lambda^{\prime} \int_{0}^{\tau} e^{-\lambda^{\prime} \mu} \sqrt{T(\tau)-T(\mu)} d \mu}
\end{array}
$$

This slope is independent of $\gamma$ and of $\sigma, V$, and $a$.

This means that two decay systems of the same age $(\tau)$ and similar diffusion histories $[D(\tau)]$ will plot on a straight line in a concordia type diagram (i.e., $r_{\lambda^{\prime}}$ plotted against $r_{\lambda}$ ). The equation of the line is 


$$
\begin{array}{r}
\frac{r_{\lambda}-e^{\lambda \tau}+1}{r_{\lambda^{\prime}}-e^{\lambda^{\prime} \tau}+1}=\frac{d r_{\lambda}}{d r_{\lambda^{\prime}}} \\
=\frac{\lambda e^{\lambda \tau} \int_{0}^{\tau} e^{-\lambda \mu} \sqrt{T(\tau)-T(\mu)} d \mu}{\lambda^{\prime} e^{\lambda^{\prime} \tau} \int_{0}^{\tau} e^{-\lambda^{\prime} \mu} \sqrt{T(\tau)-T(\mu)} d \mu}
\end{array}
$$

In the approximation $\int_{0} \tau D(\mu) d \mu \ll a^{2}$ it follows that for a given diffusion law $D(\tau)$ all grains, regardless of their geometry, will have values of $r_{\lambda}, r_{\lambda^{\prime}}$ lying on this line. The equation for $d r_{\lambda} / d r_{\lambda^{\prime}}$ given above is the asymptotic slope of the curve $r_{\lambda}, r_{\lambda}$, for a given diffusion law. A comparison of the asymptotic line with the curve for a sphere for two diffusion laws indicates that in a significant region these two solutions are very close; we therefore expect that grain geometry will not have a significant effect, except in the case of large losses ( $\sim 40$ per cent or more).

It is important to note that the diffusion coefficient only enters in the form

$$
\int_{0}^{\tau} e^{-\lambda \mu} \sqrt{T(\tau)-T(\mu)} d \mu
$$

This tends to average out fluctuations to a large extent. For example, consider the case where $D(\tau)=D_{0}[1+\cos \omega \tau]$. Then $T(\tau)=$ $D_{0}[\tau+(1 / \omega) \sin \omega \tau]$ and the slope is
For $D(\tau)=D_{1} \tau, D_{1}$ constant,

$$
\frac{d r_{\lambda}}{d r_{\lambda^{\prime}}}=\frac{\lambda e^{\lambda \tau} \sum_{m=0}^{\infty} \frac{(-1)^{m}(\lambda \tau)^{m} q_{m}}{m !(m+2)}}{\lambda^{\prime} e^{\lambda^{\prime} \tau} \sum_{m=0}^{\infty} \frac{(-1)^{m}\left(\lambda^{\prime} \tau\right)^{m} q_{m}}{m !(m+2)}}
$$

Where

$q_{m}=\int_{0}^{\pi / 2} \cos ^{m} \theta d \theta=\frac{\sqrt{\pi}}{2} \frac{\Gamma[(m+1) / 2]}{\Gamma[(m / 2)+1]}$

where $\Gamma(u)$ is the gamma function.

In general for $\mathfrak{D}(\tau)=D_{n} \tau^{n}, D_{n}$ a constant,

$$
\begin{aligned}
\frac{d r_{\lambda}}{d r_{\lambda^{\prime}}} & =\frac{\lambda e^{\lambda \tau}}{\lambda^{\prime} e^{\lambda^{\prime} \tau}} \\
& \frac{\sum_{m=0}^{\infty} \frac{(-1)^{m}(\lambda \tau)^{m}}{m !} \frac{\Gamma[m+1 / n+1]}{\Gamma[(m+1 / n+1)+3 / 2]}}{\sum_{m=0}^{\infty} \frac{(-1)^{m}\left(\lambda^{\prime} \tau\right)^{m}}{m !} \frac{\Gamma[m+1 / n+1]}{\Gamma[(m+1 / n+1)+3 / 2]}}
\end{aligned}
$$

These series converge quite rapidly and can be easily computed with a desk calculator.

\section{Appendix 2}

Theorem 2. Consider the differential equation

$$
\partial C / \partial \tau-D(\tau) \nabla^{2} C=\varphi(\mathbf{r}, \tau)
$$

$$
\frac{d r_{\lambda}}{d r_{\lambda^{\prime}}}=\frac{\lambda e^{\lambda \tau} \int_{0}^{\tau} e^{-\lambda \mu} \sqrt{\tau-\mu+(1 / \omega)(\sin \omega \tau-\sin \omega \mu)}}{\lambda^{\prime} e^{\lambda^{\prime} \tau} \int_{0}^{\tau} e^{-\lambda^{\prime} \mu} \sqrt{\tau-\mu+(1 / \omega)(\sin \omega \tau-\sin \omega \mu)}}
$$

For changes with a frequency $\omega$ that are large it is evident that the slope approaches that for the case $D(\tau)=D_{0}$ in spite of the fact that the ratio $D_{\max } / D_{\min }=\infty$. Values of $d r_{\lambda} / d r_{\lambda}$, for the above case are tabulated for different values of $\omega(\omega \equiv 2 \pi / P)$ and $\tau$. It can be seen (Table 3) that the effects are very small.

The function $d r_{\lambda} / d r_{\lambda^{\prime}}$ is shown for different times for the following different diffusion laws in Figure 8.

$$
\text { For } D(\tau)=D_{0} \text { (a constant) }
$$$$
\frac{d r_{\lambda}}{d r_{\lambda^{\prime}}}=\frac{\lambda e^{\lambda \tau}}{\lambda^{\prime} e^{\lambda^{\prime} \tau}}\left[\frac{\frac{\tau^{1 / 2}}{\lambda}-\frac{e^{-\lambda \tau}}{\lambda^{3 / 2}} \int_{0}^{\sqrt{\lambda \tau}} e^{x^{2}} d x}{\frac{\tau^{1 / 2}}{\lambda^{\prime}}-\frac{e^{-\lambda^{\prime} \tau}}{\left(\lambda^{\prime}\right)^{3 / 2}} \int_{0}^{\sqrt{\lambda^{\prime} \tau}} e^{x^{2}} d x}\right]
$$

Define

$$
T(\tau)=\int_{0}^{\tau} D(\eta) d \eta
$$

Then

$$
D(\tau)\left[(\partial / \partial T)-\nabla^{2}\right] C=\varphi(\mathrm{r}, \tau)
$$

Let $f(\mathbf{r}, \tau, \mu)$ be a solution of $\left[(\partial / \partial \tau)-\nabla^{2}\right] f=0$ such that $f(\mathbf{r}, \tau, \mu)=\varphi(\mathbf{r}, \mu)$ for $\tau \leq 0$ and that $f(\mathbf{r}, \tau, \mu)=0$ on a surface $\sigma$. Then the solution to (1) with the initial condition $C(\mathbf{r}, 0)=0$ and the boundary condition $C(\mathbf{r}, \tau)=0$ on $\sigma$ is, given by

$$
C(\mathbf{r}, \tau)=\int_{0}^{\tau} f[\mathbf{r}, T(\tau)-T(\mu), \mu] d \mu
$$


TABLE 3

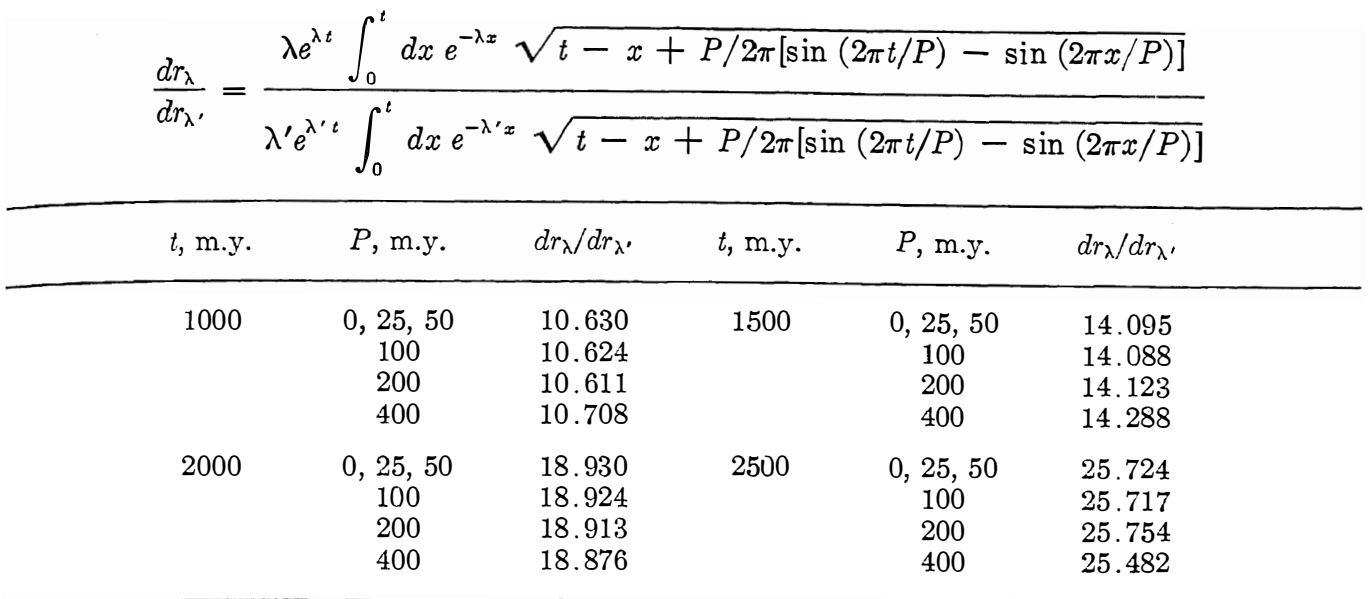

The form of theorem 2 as given above was considerably simplified as the result of a suggestion made by G. W. Wetherill (personal communication).

For the case of the simultaneous diffusion of daughter product and parent, the equations are

$$
\begin{aligned}
& \partial U_{\lambda}(\mathbf{r}, \tau) / \partial \tau \\
& \quad=D^{*}(\tau) \nabla^{2} U_{\lambda}(\mathrm{r}, \tau)-\lambda U_{\lambda}(\mathrm{r}, \tau) \\
& \frac{\partial C_{\lambda}(\mathrm{r}, \tau)}{\partial \tau} \\
& \quad=D(\tau) \nabla^{2} C_{\lambda}(\mathrm{r}, \tau)+\lambda U_{\lambda}(\mathrm{r}, \tau)
\end{aligned}
$$

Here $D(\tau)$ and $D^{*}(\tau)$ are the diffusion coefficients of daughter and parent, respectively. The boundary conditions are taken as $C_{\lambda}(\mathrm{r}, \tau)=$ $\mathrm{U}_{\lambda}(\mathrm{r}, \tau)=0$ on a surface $\sigma$. The initial conditions are $C_{\lambda}(r, 0)=0$, and $U_{\lambda}(r, 0)=U_{\lambda^{0}}$. Solutions $r_{\lambda}$ for a variety of geometries can be readily obtained. In particular, for the case of a sphere of radius $a$,

$$
\begin{aligned}
\mathrm{U}_{\lambda}(r, \tau)= & \frac{2 a}{\pi r} \mathrm{U}_{\lambda}{ }^{0} e^{-\lambda \tau} \\
& \cdot \sum_{m=1}^{\infty} \exp \left[-m^{2} \pi^{2} T^{*}(\tau) / a^{2}\right] \\
& \cdot(-1)^{m+1} \frac{\sin (m \pi r / a)}{m}
\end{aligned}
$$

where

$$
r_{\lambda}\left(\tau, \frac{D(\tau)}{a^{2}}, \frac{D^{*}(\tau)}{a^{2}}\right)=\frac{\lambda e^{\lambda \tau} \sum_{n=1}^{\infty} \frac{1}{n^{2}} \int_{0}^{\tau} \exp \left\{-n^{2} \pi^{2}[T(\tau)-T(\mu)] / a^{2}-\lambda \mu-n^{2} \pi^{2} T^{*}(\mu) / a^{2}\right\} d \mu}{\sum_{m=1}^{\infty} \frac{\exp \left[-m^{2} \pi^{2} T^{*}(\tau) / a^{2}\right]}{m^{2}}}
$$

$$
T^{*}(\tau)=\int_{0}^{\tau} D^{*}(\eta) d \eta
$$

$\int_{0}^{a} 4 \pi r^{2} U_{\lambda}(r, \tau) d r=\frac{8 a^{3}}{\pi} U_{\lambda}^{0} e^{-\lambda \tau}$

$$
\cdot \sum_{m=1}^{\infty} \frac{\exp \left[-m^{2} \pi^{2} T^{*}(\tau) / a^{2}\right]}{m^{2}}
$$

Using theorem 2 and equation 3 [Carslaw and Jaeger, 1959, p. 233] we obtain

$$
\begin{gathered}
C_{\lambda}(r, \tau)=\frac{2 a \lambda U_{\lambda}^{0}}{\pi r} \sum_{n=1}^{\infty} \frac{\sin (n \pi r / a)}{n}(-1)^{n+1} \\
\cdot \int_{0}^{r} \exp \left\{-n^{2} \pi^{2}[T(\tau)-T(\mu)] / a^{2}\right. \\
\left.-\lambda_{\mu}-n^{2} \pi^{2} T^{*}(\mu) / a^{2}\right\} d \mu
\end{gathered}
$$

$$
\int_{0}^{a} 4 \pi r^{2} C_{\lambda}(r, \tau) d r=\frac{8 a^{3} \lambda U_{\lambda}^{0}}{\pi} \sum_{n=1}^{\infty} \frac{1}{n^{2}}
$$

$$
\cdot \int_{0}^{\tau} \exp \left\{-n^{2} \pi^{2}[T(\tau)-T(\mu)] / a^{2}\right.
$$$$
\left.-\lambda \mu-n^{2} \pi^{2} T^{*}(\mu) / a^{2}\right\} d \mu
$$ 
When $D^{*}(\tau)=D(\tau)$ for all $\tau, r_{\lambda}=e^{\lambda \tau}-1$. When the diffusion coefficients are the same, therefore, the resulting ages will be concordant.

The case $D(\tau)=D_{0}, D^{*}(\tau)=D_{0}{ }^{*}$ and the case where $D_{0}$ and $D_{0}^{*}$ are increased by factors $K$ and $K^{*}$ during a pulse of metamorphism of finite duration were treated previously by Wetherill [1963].

For the case of a single episodic loss at time $\tau_{1}$ years ago for a primary event $\tau$ years ago,

$$
\begin{gathered}
D(\mu)=u \delta\left(\mu-\tau+\tau_{1}\right) \\
D^{*}(\mu)=u^{*} \delta\left(\mu-\tau+\tau_{1}\right) \\
r_{\lambda}=g\left(u / a^{2}\right) / g\left(u^{*} / a^{2}\right) e^{\lambda \tau} \\
-g\left(u / a^{2}\right) / g\left(u^{*} / a^{2}\right) e^{\lambda_{1}}+e^{\lambda \tau_{1}}-1
\end{gathered}
$$

where

$$
g(x)=\sum_{n=1}^{\infty} \frac{1}{n^{2}} e^{-n^{2} x^{2} x}
$$

The function $g(x)$ is tabulated in Table 4 .

If the diffusion coefficient is dominated by saturation radiation damage defects or if the

TABLE 4

$$
\frac{g(x)}{\sum_{1}^{\infty} 1 / n^{2}}=\frac{6}{\pi^{2}} \sum_{n=1}^{\infty} \frac{e^{-n^{2} x^{3} x}}{n^{2}}
$$

\begin{tabular}{lccc}
\hline \multicolumn{1}{c}{$x$} & $6 g(x) / \pi^{2}$ & $x$ & $6 g(x) / \pi^{2}$ \\
\hline 0 & 0.99989 & 0.088 & 0.25980 \\
0.002 & 0.85461 & 0.098 & 0.23428 \\
0.004 & 0.79790 & 0.108 & 0.21152 \\
0.006 & 0.75578 & 0.114 & 0.19902 \\
0.008 & 0.72122 & 0.124 & 0.17993 \\
0.010 & 0.69148 & 0.130 & 0.16941 \\
0.012 & 0.66517 & 0.136 & 0.15953 \\
0.016 & 0.61981 & 0.142 & 0.15025 \\
0.020 & 0.58126 & 0.148 & 0.14152 \\
0.024 & 0.54757 & 0.156 & 0.13069 \\
0.028 & 0.51755 & 0.164 & 0.12070 \\
0.032 & 0.49044 & 0.172 & 0.11149 \\
0.036 & 0.46571 & 0.182 & 0.10098 \\
0.040 & 0.44297 & 0.192 & 0.091463 \\
0.044 & 0.42192 & 0.206 & 0.079637 \\
0.048 & 0.40235 & 0.220 & 0.069346 \\
0.052 & 0.38407 & 0.236 & 0.059208 \\
0.058 & 0.35875 & 0.252 & 0.050554 \\
0.062 & 0.34310 & 0.276 & 0.039889 \\
0.068 & 0.32126 & 0.304 & 0.030257 \\
0.074 & 0.30114 & 0.346 & 0.019989 \\
0.078 & 0.28858 & 0.400 & 0.011730 \\
\hline
\end{tabular}

number of defects are (U) impurity related, this corresponds to the arguments previously made for $D_{0} \propto \mathrm{U}_{\lambda}$. Hence $u / a^{2}$ and $u^{*} / a^{2}$ are then proportional to $\mathrm{U}_{\lambda}$.

The function $f=r_{\lambda} /\left(e^{\lambda \tau}-1\right)$ can be calcu. lated from the above equation. However, for the episodic model it is more convenient to define the quantity $R=l_{1} / L_{1}$ after Wetherill [1956]. It follows that from the preceding equations $R=g\left(u / a^{2}\right) / g\left(u^{*} / a^{2}\right)$ and is independent of $\lambda$. $R$ is the fractional distance (in units of $L_{1}$ ) along the chord connecting the points $\tau$ and $\tau_{1}$ on the concordia curve. $R=1$ for $u=u^{*}$. This equation relates the fractional distance on the episodic loss chord to diffusion parameters instead of the generalized loss function as used by Wetherill in his homogeneous system (box model) theory.

Asymptotic theory. For the asymptotic case, consider the one-dimensional problem. For the parent $U_{\lambda}$

$$
\begin{gathered}
\frac{\partial \mathrm{U}_{\lambda}(x, \tau)}{\partial \tau}=D^{*}(\tau) \frac{\partial^{2}}{\partial x^{2}} \mathrm{U}_{\lambda}(x, \tau)-\lambda \mathrm{U}_{\lambda}(x, \tau) \\
\mathrm{U}_{\lambda}(x, 0)=\mathrm{U}_{\lambda}{ }^{0} \quad \mathrm{U}_{\lambda}(0, \tau)=0 \\
T^{*}(\tau)=\int_{0}^{\tau} D^{*}(\eta) d \eta \\
\mathrm{U}_{\lambda}(x, \tau)=\mathrm{U}_{\lambda}{ }^{0} \operatorname{erf}\left\{x / 2 \sqrt{T^{*}(\tau)}\right\} e^{-\lambda \tau}
\end{gathered}
$$

The net flux of parent through the surface

$$
\begin{aligned}
Q_{\lambda}{ }^{*}=\frac{2 \sigma}{\sqrt{\pi}} \mathrm{U}_{\lambda}{ }^{0}[ & \sqrt{T^{*}(\tau)} e^{-\lambda \tau} \\
& \left.+\lambda \int_{0}^{\lambda} \sqrt{T^{*}(\eta)} e^{-\lambda \eta} d \eta\right],
\end{aligned}
$$

The net number of parent atoms that would have been in the system at time $\tau$ but that were lost by diffusion is

$$
N=\frac{\sigma \mathrm{U}_{\lambda}^{0} 2 e^{-\lambda \tau} \sqrt{T^{*}(\tau)}}{\sqrt{\pi}}
$$

The net number of decays that would have occurred inside the system but that were lost because of parent diffusion is

$$
B=\frac{2 \mathrm{U}_{\lambda}^{0}}{\pi} \sigma \lambda \int_{0}^{\tau} e^{-\lambda \eta} \sqrt{T^{*}(\eta)} d \eta
$$

For the diffusion of the daughter in the onedimensional case, we have, using $\mathrm{U}_{\lambda}(x, \tau)$, 
theorem 2, and equation 1 [Carslaw and Jaeger, 1959, p. 59]

$$
\begin{gathered}
C_{\lambda}(x, \tau)=\lambda \mathrm{U}_{\lambda}^{0} \int_{0}^{\tau} \frac{d \mu e^{-\lambda \mu}}{2 \sqrt{\pi}[T(\tau)-T(\mu)]^{1 / 2}} \\
\cdot \int_{0}^{\infty} \operatorname{erf}\left\{\frac{x^{\prime}}{2 \sqrt{T^{*}(\mu)}}\right\} e^{-\left(x-x^{\prime}\right)^{2 / 4}[\mathrm{~T}(\tau)-\mathrm{T}(\mu)]} \\
\left.-e^{-\left(x+x^{\prime}\right)^{2 / 4}[\mathrm{~T}(\tau)-\mathrm{T}(\mu)]}\right\} d x^{\prime} \\
C_{\lambda}(x, 0)=0 \quad C_{\lambda}(0, \tau)=0
\end{gathered}
$$

The net flux $Q_{\lambda}(\tau)$ through the surface $x=0$ can be written in the following form after some manipulation analogous to the treatment in appendix 1:

$$
\begin{aligned}
Q_{\lambda}(\tau)= & \lambda \mathrm{U}_{\lambda}^{0} \frac{2 \sigma}{\sqrt{\pi}} \\
& \cdot\left[\int_{0}^{\tau} d \mu e^{-\lambda \mu} \sqrt{T^{*}(\mu)+T(\tau)-T(\mu)}\right. \\
& \left.-\int_{0}^{\tau} d \mu e^{-\lambda \mu} \sqrt{T^{*}(\mu)}\right]
\end{aligned}
$$

For a grain of volume $V$ and surface area $\sigma$ and effective radius of curvature $a$, if

$$
\int_{0}^{\tau} D^{*}(\eta) d \eta \ll a^{2}
$$

and

$$
\begin{gathered}
\int_{0}^{\tau} D(\eta) d \eta \ll a^{2} \\
r_{\lambda}=\frac{V U_{\lambda}^{0}\left(1-e^{-\lambda \tau}\right)-Q_{\lambda}-B}{V U_{\lambda}^{0} e^{-\lambda \tau}-N}
\end{gathered}
$$

$$
r_{\lambda}=\frac{e^{\lambda \tau}-1-\frac{2 \sigma \lambda e^{\lambda \tau}}{\sqrt{\pi} V} \int_{0}^{\tau} d \mu e^{-\lambda \mu} \sqrt{T^{*}(\mu)+T(\tau)-T(\mu)}}{1-(2 \sigma / \sqrt{\pi} V) \sqrt{T^{*}(\tau)}}
$$

For $T^{*}(\tau) \equiv 0$ we obtain the equation for no diffusion of parent. For $T(\tau) \equiv T^{*}(\tau) r_{\lambda}=e^{\lambda \tau}-1$.

For the case of no daughter loss $\left[T(\tau) \equiv 0\right.$ ]. Writing $\gamma^{2} T^{*}(\eta)$ for $T^{*}(\eta)$, where $\gamma$ is a dimensionless parameter,

$$
\frac{d r_{\lambda} / d \gamma}{d r_{\lambda^{\prime}} / d \gamma}=\frac{\left(e^{\lambda \tau}-1\right) \sqrt{T^{*}(\tau)}-\lambda e^{\lambda \tau} \int_{0}^{\tau} d \mu e^{-\lambda \mu} \sqrt{T^{*}(\mu)}}{\left(e^{\lambda^{\prime} \tau}-[1) \sqrt{T^{*}(\tau)}-\lambda^{\prime} e^{\lambda^{\prime} \tau} \int_{0}^{\tau} d \mu e^{-\lambda^{\prime} \mu} \sqrt{T^{*}(\mu)}\right.}
$$

so that for a fixed $\tau$ the trajectory of parent loss for $\int_{0}{ }^{\top} D^{*}(\eta) d \eta \ll a^{2}$ is a straight line passing through the point on concordia, the equation being

$$
\frac{r_{\lambda}\left[\tau, \gamma D^{*}(\tau)\right]-e^{\lambda \tau}+1}{r_{\lambda^{\prime}}\left[t, \gamma D^{*}(\tau)\right]-\lambda^{\lambda^{\prime}}+1}=\frac{\left(e^{\lambda \tau}-1\right) \sqrt{T^{*}}(\tau)-\lambda e^{\lambda \tau} \int_{0}^{\tau} d \mu e^{-\lambda \mu} \sqrt{T^{*}(\mu)}}{\left(e^{\lambda^{\prime} \tau}-1\right) \sqrt{T^{*}(\tau)}-\lambda^{\prime} e^{\lambda^{\prime} \tau} \int_{0}^{\tau} d \mu e^{-\lambda^{\prime} \mu} \sqrt{T^{*}(\mu)}}
$$

Since $T^{*}(\tau)$ is a monotonically increasing function, it follows that $r_{\lambda}$ and $r_{\lambda^{\prime}}$ will lie on a straight line extending above the concordia curve.

The slope of this line will be different for the case of pure daughter diffusion with the identical form for $D(\tau)$.

For the case of simultaneous diffusion of parent and daughter for a system of samples that have the same ratio of $T^{*}(\mu) / T(\mu)$ for all $\mu \leq \tau, r_{\lambda}, r_{\lambda^{\prime}}$ lie on straight line of slope

$$
\frac{d r_{\lambda}}{d r_{\lambda^{\prime}}}=\frac{\left(e^{\lambda \tau}-1\right) \sqrt{T^{*}(\tau)}-\lambda e^{\lambda \tau} \int_{0}^{\tau} d \mu e^{-\lambda \mu} \sqrt{T^{*}(\mu)+T(\tau)-T(\mu)}}{\left(e^{\lambda^{\prime} \tau}-1\right) \sqrt{T^{*}(\tau)}-\lambda^{\prime} e^{\lambda^{\prime} \tau} \int_{0}^{\tau} d \mu e^{-\lambda^{\prime} \mu} \sqrt{T^{*}(\mu)+T(\tau)-T(\mu)}}
$$


This corresponds to the case of a suite of minerals for which $T(\tau)=\gamma t(\tau)$ and $T^{*}(\tau)=\gamma^{*} t(\tau)$ where $\gamma$ and $\gamma^{*}$ are constants for each particular sample. The values for $d r_{\lambda} / d r_{\lambda}$, for various ratios $k \equiv \gamma^{*} / \gamma$ and different times for $t(\tau)=\tau$ and $\tau^{2}$ are shown in Figure 11. The slope for pure lead diffusion is greater than the slope for pure uranium diffusion with the same diffusion law. As the uranium diffusion increases from zero, the asymptotic line decreases in slope until it reaches the limiting slope for pure uranium loss. It is evident that the change in slope with increasing uranium diffusion is very rapid at first and then changes relatively slightly with increasing $k$. This effect, first found by Wetherill (personal communication), appears to be independent of the diffusion law.

Acknowledgments. I would like to acknowledge several valuable discussions with $\mathrm{G}$. W. Wetherill, L. T. Silver, P. Gast, W. B. Kamb, and G. J. F. Tilton. I a m particularly indebted to R. F. Christy, who generously aided by programming some of the solutions. Mr. A. Dahlen performed many of the computations of the asymptotic forms. Tabulated values for the constant diffusion coefficient case were provided by G. L. Davis.

This work was supported by a grant from the National Science Foundation and a grant from the American Chemical Society Petroleum Research Fund.

\section{ReFERENCES}

Carslaw, H. S., and J. C. Jaeger, Conduction of Heat in Solids, 2nd edition, p. 32, Oxford University Press, London, 1959.

Crank, J., The Mathematics of Diffusion, Oxford University Press, London, 1956.

Dienes, G. J., and A. C. Damask, Radiation enhanced diffusion in solids, J. Appl. Phys., 29, 1713, 1958.

Dienes, G. J., and A. C. Damask, Kinetics of vacancy-interstitial annihilation, 1 and 2, Phys, Rev., 125, 449, 1962.

Hurley, P. M., The He age method, in Nuclear
Geology, edited by H. Faul, John Wiley \& Sons, New York, 1954.

Kuovo, O., Radioactive age of some Finnish Procambrian minerals, Bull. Comm. Geol. Finland, 182, 1958.

Lanphere, M. A., Geochronologic studies in the eastern Mojave Desert, California, J. Geol., in press, 1963.

Nicolaysen, L. O., Solid diffusion radioactive minerals and the measurement of absolute geologic age, Geochim. Cosmochim. Acta, 11, 41, 1957.

Russell, R. D., and L. H. Ahrens, Additional regularities among discordant lead-uranium ages, Geochim. Cosmochim. Acta, 11, 213-218, 1957.

Silver, L. T., Nuclear Geophysics, Proc. Woods Hole Conf. Nuclear Probl. Geol., Natl. Acad. Sci., U. S., Publ. 1075, 1962.

Silver, L. T., and S. Deutsch, Uranium lead method on zircons, Proc. N. Y. Acad. Sci., 91, 279-283, 1961.

Silver, L. T., and C. R. McKinney, U-Pb isotopic age studies of a Precambrian granite, Marble Mountains, Calif. (abstract), Bull. Geol. Soc. Am., Cordilleran Section Meeting, 1962.

Silver, L. T., C. R. McKinney, S. Deutsch, and J. Bolinger, Precambrian age determinations in the western San Gabriel Mountains, Calif., J. Geol., 71, 196-214, 1963.

Tilton, G. R., Volume diffusion as a mechanism for discordant lead ages, J. Geophys. Res., 65, 2933-2945, 1960.

Wasserburg, G. J., $\mathrm{A}^{40}-\mathrm{K}^{40}$ dating, in Nuclear Geol ogy, edited by H. Faul, John Wiley \& Sons, New York, 1954.

Wasserburg, G. J., Nuclear Geophysics, Proc. Woods Hole Conf. Nuclear Probl. Geol., Natl. Acad. Sci., U. S. Publ. 1075, 1962.

Wetherill, G. W., Discordant uranium lead ages, Trans. Am. Geophys. Union, 87, 320-326, 1956.

Wetherill, G. W., Discordant uranium lead ages, 2, J. Geophys. Res., 68, 2957-2965, 1963.

Holland and Gottfried, The effect of nuclear radiation on the structure of zircon, Acta Cryst., 8, 291-300, 1955.

Wasserburg, G. J., G. W. Wetherill, L. T. Silver, and P. T. Flawn, A study of the ages of the Precambrian of Texas, J. Geophys. Res., 67, 4021-4047, 1962.

(Manuscript received February 26, 1963, revised May 8, 1963.) 